FLÁVIA ANDREA KREPEL FORONDA

\title{
Duração da ventilação mecânica em pediatria: impacto da introdução de avaliações diárias e teste de respiração espontânea
}

Tese apresentada à Faculdade de Medicina da Universidade de São Paulo para obtenção do título de Doutor em Ciências

Programa de Pediatria

Orientador: Prof. Dr. Eduardo Juan Troster

São Paulo

2013 
Dados Internacionais de Catalogação na Publicação (CIP)

Preparada pela Biblioteca da

Faculdade de Medicina da Universidade de São Paulo

(C)reprodução autorizada pelo autor

Foronda, Flávia Andrea Krepel

Duração da ventilação mecânica em pediatria : impacto da introdução de avaliações diárias e teste de respiração espontânea / Flávia Andrea Krepel Foronda. -- São Paulo, 2013.

Tese(doutorado)--Faculdade de Medicina da Universidade de São Paulo.

Programa de Pediatria.

Orientador: Eduardo Juan Troster.

Descritores: 1.Ensaio clínico controlado aleatório 2.Unidade de terapia intensiva 3.Criança 4.Respiração artificial 5.Insuficiência respiratória 6.Desmame do respirador

USP/FM/DBD-019/13 
Dedicatória 
"Para realizar grandes conquistas, devemos não apenas agir, mas também sonhar; não apenas planejar, mas também acreditar."

\section{Anatole France}

Dedico essa tese ao meu marido Gú e aos meus filhos Mazi e Quito, razão da minha vida, que sempre me deram amor incondicional e aceitaram, sem reclamar, minha ausência e, principalmente, meus dias ruins.

Aos meus pais, Zélia e Pedro que sempre investiram e se dedicaram para que eu fosse não só uma boa médica mas, principalmente uma boa pessoa.

Aos meus irmãos João Gilberto e João Paulo que sempre torceram por mim e minha irmã Fabiana que esteve sempre pronta a me ajudar com gráficos, figuras e desenhos.

A minha avó Margarida que ainda hoje me ensina a viver e não desistir dos meus objetivos.

Aos meus sogros Annette e Antonio que sempre me incentivaram a seguir em frente.

Enfim, todos aqueles que acreditaram nos meus sonhos e me apoiaram nos momentos mais difíceis. 
Agradecimentos 
Pediatria... Residência de pediatria... iniciei o estágio de terapia intensiva e conheci a Lucília Santana Faria, então me encantei pela sua energia, sua vontade de trabalhar, sua paixão pela profissão, e todo seu conhecimento na área que eu acabaria por escolher como minha linha de pesquisa, a ventilação mecânica. A sua alegria e dedicação foram me conquistando até não ter mais volta....

Outra pessoa que me introduziu ao mundo da ventilação foi o Albert Bousso. É incrível a cabeça e também coração... que ele tem.

Meu chefe da UTI na época, era uma pessoa com uma grande capacidade de envolver e estimular as pessoas a estudarem mais profundamente aquilo do que mais gostavam. Um grande agregador, sempre disposto a ajudar no crescimento profissional e pessoal dos colegas. Devagar, sempre respeitando todos os meus momentos de vida, foi me convencendo a entrar nessa empreitada. Foi assim que o Eduardo Juan Troster me colocou na pós graduação. Manteve sua postura em todo o meu caminho, sempre presente, sempre disposto a uma conversa, a uma discussão. Mais que tudo foi um amigo me incentivando nos momentos difíceis.

Tive a grande sorte de que o chefe que sucedeu o Troster continuou me apoiando de forma incondicional e valorizou todo o meu esforço me ajudando, sempre que possível. Assim, tenho muito a agradecer ao Artur Figueiredo Delgado.

Resolvi estudar mais profundamente desmame da ventilação mecânica, que na época ainda não era muito valorizado na pediatria e li vários artigos de um tal Julio Farias. Achei que antes de começar a tese poderia conhecer um pouco mais o seu trabalho. Através do Troster consegui organizar um estágio de algumas semanas no seu serviço, no Hospital de Niños, em Buenos Aires. Fui recebida de braços abertos!! Uma pessoa maravilhosa, sempre pronto a dividir todo seu conhecimento e experiência. Na Argentina, vi como eles realizavam desmame e pude criar uma base sólida para começar meu estudo. Em vários momentos do meu estudo, me reportei, ou tive a chance de encontrá-lo pessoalmente, sempre com alguma contribuição importante para meu trabalho. Quando meu trabalho ficou pronto foi um dos sorrisos contagiantes que recebi. Infelizmente não está mais aqui entre nós, mas com certeza está feliz pela minha realização.

No andamento do trabalho a coleta de dados foi uma das missões mais difíceis e trabalhosa. Nesta missão tive a ajuda de todos os Residentes de Terapia Intensiva e os Residentes de Pediatria, passando no Hospital Universitário e no Instituto da Criança, sem essa ajuda não conseguiria completar minha casuística. Em particular, queria agradecer ao Daniel Luis Assumpção Pitarello, preceptor dos residentes, pela dedicação e ajuda fundamental nesta coleta de dados. 
A ajuda e compreensão de todos os médicos e amigos das Equipes das UTIs do ICr e do HU foi muito importante. Sem eles, que tiveram a árdua tarefa diária de manter o protocolo em andamento (Não é Fácil!!), não conseguiria ter ido tão longe. Não posso deixar de mencionar também as equipes de enfermagem e fisioterapia, que tanto se dedicam aos pacientes internados e que me ajudaram muito na realização do protocolo.

Gostaria de agradecer especialmente a minha amiga Flavia Panico Rossi pelas gargalhadas, por compartilhar as dificuldades e a paixão pela ventilação mecânica e ter a incrível capacidade de fazer o ruim parecer menos pior.

Mas o trabalho não acabou por aí. Começou então a fase da publicação... e aqui são incontáveis as pessoas que me ajudaram e que gostaria de agradecer!! Se eu esquecer alguém, por favor me perdoe!! Começando pela Carmen Silvia Valente Barbas. Essa mulher é impressionante! É incrível a capacidade e dedicação que ela tem! Me ajudou muito, sempre revendo todos os meus manuscritos, ajudando nas respostas aos revisores (foram no total 5 idas e vindas) além, de estar sempre disponível para qualquer dúvida ou necessidade.

Nessa fase também não posso deixar de mencionar a equipe da Dendrix, que me ajudou na tradução para o inglês, na formatação do manuscrito e todas as respostas para revisores.

O Alexandre Archanjo Ferraro foi muito importante pela experiência compartilhada em epidemiologia e o Rogério Prado Ruscitto foi fundamental para todas as análises estatísticas. O Rogério é uma pessoa extremamente objetiva e didática que conseguiu transformar o complexo em simples.

Outra pessoa que me ajudou bastante foi o Luiz Vicente Ribeiro Ferreira da Silva que com toda a paciência, sem receber nada em troca, leu a última versão do meu artigo e me deu sugestões totalmente pertinentes. $O$ Eduardo Correa Meyer também leu minha tese e deu as últimas sugestões antes de eu ter coragem de mandar imprimir.

Mas, gostaria de agradecer principalmente, todos os pacientes e familiares que participaram do meu estudo. Sem eles, todo esse trabalho não teria o menor sentido... Afinal é para tentar cuidar deles um pouquinho melhor que tudo isso começou. Enfim...

OBRIGADA!!! Lú, Albert, Troster, Artur, Julio, residentes, Daniel, meus amigos médicos das UTIs do $\mathrm{ICr}$ e HU, enfermeiras, fisioterapeutas, Flá, Carmenzita, Dendrix, Alexandre, Rogério, Vic, Edu e principalmente todos os pacientes e familiares que permitiram que esse trabalho fosse realizado. 
Esta tese está de acordo com as seguintes normas, em vigor no momento desta publicação:

Referências: adaptado de International Committee of Medical Journals Editors (Vancouver)

Universidade de São Paulo. Faculdade de Medicina. Serviço de Biblioteca e Documentação. Guia de apresentação de dissertações, teses e monografias. Elaborado por Anneliese Carneiro da Cunha, Maria Julia de A. L. Freddi, Maria F. Crestana, Marinalva de Souza Aragão, Suely Campos Cardoso, Valéria Vilhena. $2^{\mathrm{a}}$ ed. São Paulo: Serviço de Biblioteca e Documentação; 2005.

Abreviaturas dos títulos dos periódicos de acordo com List of Journals Indexed in Index Medicus. 
Sumário 


\section{SUMÁRIO}

Lista de Siglas

Lista de Figuras

Lista de Tabelas

Resumo

Abstract

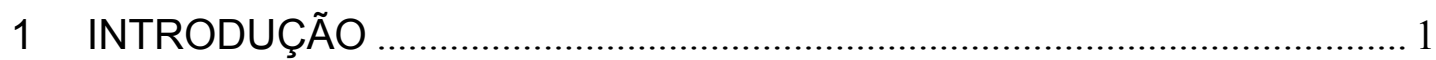

1.1 Insuficiência Respiratória Aguda ........................................................ 2

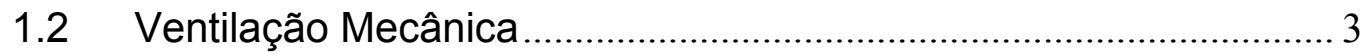

1.3 Desmame da Ventilação Mecânica ..................................................... 4

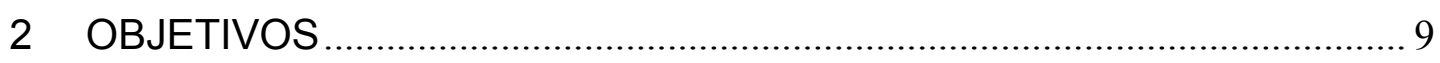

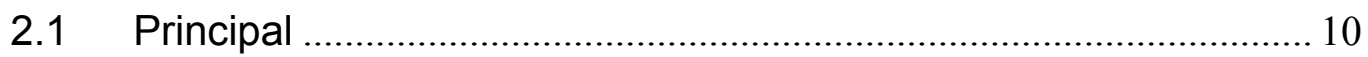

2.2 Secundários ............................................................................ 10

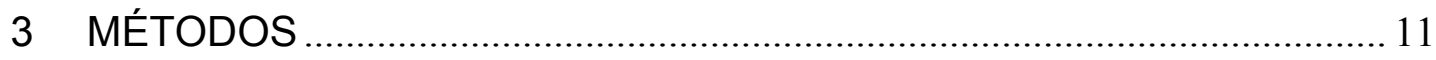

3.1 Desenho do estudo ......................................................................... 13

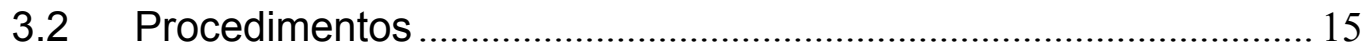

3.3 Desmame e extubação................................................................... 18

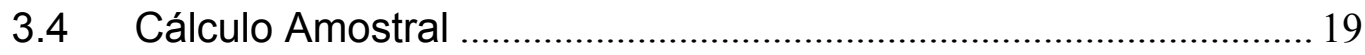

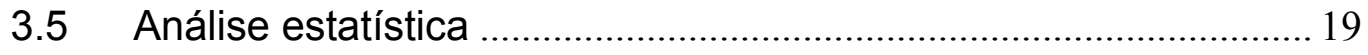

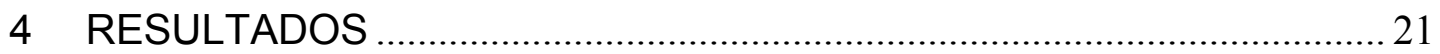

4.1 Características gerais dos pacientes .............................................. 22

4.2 Duração da ventilação mecânica......................................................... 28

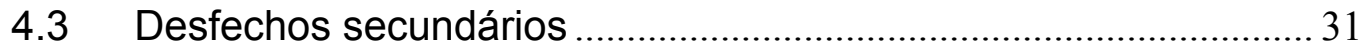

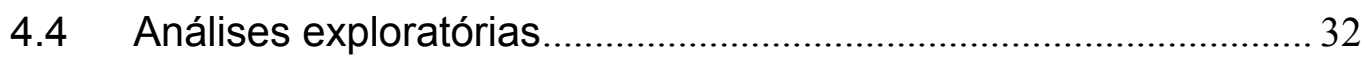

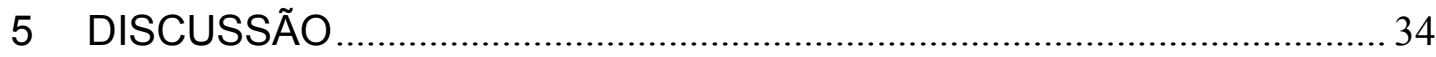

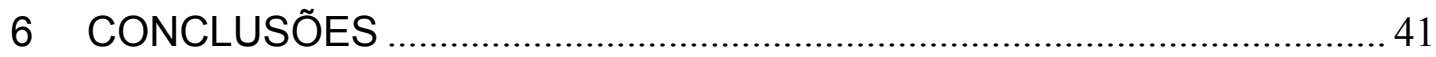

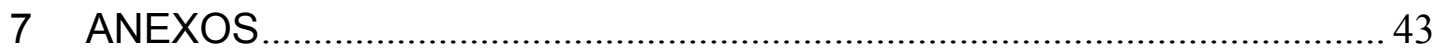

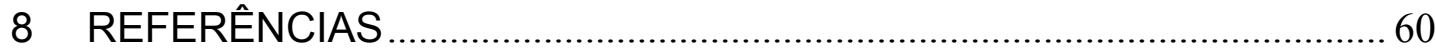


Listas 


\section{Siglas}

TRE: Teste de Respiração Espontânea

VM: Ventilação Mecânica

VNI: Ventilação não Invasiva

cm $\mathrm{H}_{2} \mathrm{O}$ : centímetros de água

PEEP: Pressão Expiratória Final Positiva

PS: Pressão de Suporte

PI: Pressão Inspiratória

$\mathrm{FiO}_{2}$ : Fração inspirada de Oxigênio

IC: Intervalo de Confiança

UTIp: Unidade de Terapia Intensiva pediátrica

OVAS: Obstrução de Vias Aéreas Superiores

mcg/kg/min: microgramas por kilograma por minuto

g/dL: gramas por decilitro

FR: Frequência Respiratória

FC: Frequência Cardíaca

PA: Pressão Arterial

SaO2: Saturação arterial de Oxigênio

PaCO2: Pressão arterial de Gás Carbônico

pH: potencial Hidrogeniônico

PIM 2: Índice Pediátrico de Mortalidade 2

PRISM: Risco de Mortalidade Pediátrico

SDRA: Síndrome do Desconforto Respiratório Agudo

OMS: Organização Mundial de Saúde

IMC: Índice de Massa Corpórea 


\section{Figuras}

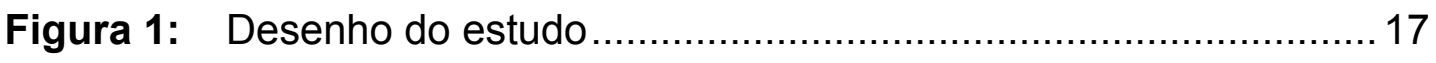

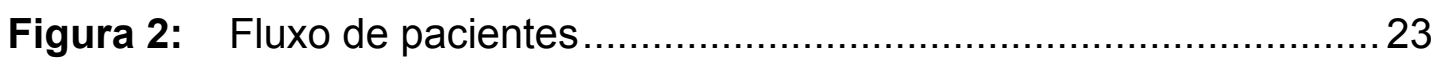

Figura 3: Comparação do tempo $p /$ extubação entre os grupos.................29 


\section{Tabelas}

Tabela 1 - Características clínicas e demográficas dos pacientes randomizados de acordo com o grupo de estudo.

Tabela 2 - Características clínicas e demográficas dos pacientes analisados de acordo com o grupo de estudo

Tabela 3 - Comparação dos grupos quanto ao estado nutricional

Tabela 4 - Análise multivariada dos fatores preditivos do tempo para extubação

Tabela 5 - Complicações respiratórias nos dois grupos

Tabela 6 - Comparação dos parâmetros ventilatórios antes da extubação nos dois grupos 
Resumo 
Foronda FAK. Duração da ventilação mecânica em pediatria: impacto da introdução de avaliações diárias e teste de respiração espontânea [Tese] . São Paulo: Faculdade de Medicina, Universidade de São Paulo; 2013.

Introdução: Ventilação mecânica (VM) é frequente em crianças com insuficiência respiratória aguda e está associada a complicações, sendo, portanto, necessários esforços para encurtar a sua duração. Objetivos: Verificar se a realização de avaliações diárias e testes de respiração espontânea (TRE), poderiam encurtar a duração da VM em crianças, quando comparado ao desmame habitualmente realizado em pediatria. Objetivos secundários incluíram a avaliação da taxa de falha de extubação e a necessidade de ventilação não invasiva (VNI). Desenho: Estudo prospectivo, randomizado, controlado. Ambiente: Duas unidades de cuidados intensivos pediátricos, em Hospitais Universitários no Brasil. Pacientes: Crianças entre 28 dias e 15 anos de idade que estavam recebendo VM por pelo menos 24 horas no período de julho 2007 a julho 2009. Intervenções: Os pacientes foram aleatoriamente designados para um dos dois protocolos de desmame. No grupo de teste, as crianças foram submetidas a avaliações diárias para verificar prontidão para desmame, depois foi realizado TRE com pressão de suporte de $10 \mathrm{~cm}$ H2O e PEEP de $5 \mathrm{~cm} \mathrm{H} 2 \mathrm{O}$ por um período de 2 horas. O TRE foi repetido no dia seguinte nas crianças que não passaram no primeiro teste. No grupo controle, o desmame foi realizado por critérios clínicos e laboratoriais como de rotina nas unidades. Resultados: 294 crianças elegíveis foram randomizadas, sendo 155 para o grupo de teste e 139 para o grupo controle. O tempo de VM foi menor no grupo de teste onde a duração mediana foi de 3,5 dias (95\% intervalo de confiança [IC]: 3,0-4,0) em comparação com o grupo controle onde a mediana do tempo de VM foi de 4,7 dias (95\% IC: 4,1-5,3) $(p=0,013)$. Esta redução significativa na duração da VM não foi associada a um aumento das taxas de falha de extubação ou VNI e representa uma redução de $30 \%$ no risco de permanecer em VM (Risco Relativo: 0,70). Conclusões: A avaliação diária para verificar a prontidão para o desmame combinada ao TRE reduziu a duração de VM em crianças, sem aumentar a taxa de falha de extubação ou a necessidade de VNI.

Descritores: 1.Ensaio clínico controlado aleatório 2.Unidade de terapia intensiva 3.Criança 4.Respiração artificial 5.Insuficiência respiratória 6.Desmame do respirador 
Summary 
Foronda FAK. The impact of daily evaluation and spontaneous breathing test on the duration of pediatric mechanical ventilation: $A$ randomized controlled trial [Thesis]. São Paulo: "Faculdade de Medicina, Universidade de São Paulo"; 2013.

Introduction: Mechanical ventilation (MV) is common in children with acute respiratory failure and is associated with several complications requiring efforts to shorten its duration. Objectives: To assess whether the combination of daily evaluation and use of a spontaneous breathing test (SBT) could shorten the duration of mechanical ventilation as compared with weaning based on our standard of care. Secondary outcome measures included extubation failure rate and the need for noninvasive ventilation (NIV).Design: A prospective, randomized, controlled trial. Setting: Two pediatric intensive care units at university hospitals in Brazil. Patients: The trial involved children between 28 days and 15 years of age who were receiving MV for at least 24 hours. Interventions: Patients were randomly assigned to one of two weaning protocols. In the test group, the children underwent a daily evaluation to check readiness for weaning and then submitted to an SBT with $10 \mathrm{~cm} \mathrm{H}_{2} \mathrm{O}$ pressure support and a PEEP of $5 \mathrm{~cm}$ $\mathrm{H}_{2} \mathrm{O}$ for 2 hours. The SBT was repeated the next day for children who failed it. In the control group, weaning was performed according to standard care procedures. Measurements and main results: A total of 294 eligible children were randomized, with 155 to the test group and 139 to the control group. The time to extubation was shorter in the test group, where the median MV duration was 3.5 days ( $95 \%$ confidence interval [Cl], 3.0 to 4.0 ) as compared to 4.7 days $(95 \% \mathrm{Cl}, 4.1$ to 5.3$)$ in the control group $(p=0.0127)$. This significant reduction in the MV duration for the intervention group was not associated with increased rates of extubation failure or NIV. It represents a $30 \%$ reduction in the risk of remaining on MV (hazard ratio: 0.70 ). Conclusions: A daily evaluation to check readiness for weaning combined with a SBT reduced the MV duration for children on MV for more than 24 hours, without increasing the extubation failure rate or the need for NIV.

Descriptors: 1.Randomized controlled trial 2.Intensive care units 3.Children 4.Respiration, artificial 5.Respiratory insufficiency 6.Ventilator weaning 
1 INTRODUÇÃO 


\subsection{Insuficiência Respiratória Aguda}

A insuficiência respiratória aguda na infância é muito frequente sendo a principal causa de óbito em crianças menores de 5 anos. Cerca de 2 a 3 milhões de crianças morrem por ano, no mundo, em decorrência de problemas respiratórios. ${ }^{1}$

Essa alta suscetibilidade para desenvolver falência respiratória pode ser explicada pelo fato das crianças possuírem uma via aérea menor, uma demanda metabólica maior, uma reserva respiratória menor e mecanismos compensatórios inadequados, o que pode levar a uma evolução mais rápida para a fadiga. $^{2}$

Por isso, nas crianças, a insuficiência respiratória pode rapidamente progredir para falência respiratória sendo a principal causa de parada cardíaca. Pode-se evitar essa rápida progressão através de um reconhecimento e tratamento precoce da insuficiência respiratória. ${ }^{3}$ Nesse contexto surge a ventilação mecânica como importante terapêutica coadjuvante. 


\subsection{Ventilação Mecânica}

A principal causa de internação em unidade de terapia intensiva pediátrica é a necessidade de suporte respiratório. No Centro de Terapia Intensiva do Instituto da Criança cerca de $60 \%$ dos pacientes necessitam de ventilação mecânica durante a internação. ${ }^{4}$ Os objetivos principais da ventilação mecânica são diminuir o trabalho respiratório e reverter hipoxemia grave ou acidose respiratória aguda e progressiva. As indicações de ventilação mecânica incluem não somente as doenças em que ocorre um agravo pulmonar primário como na Síndrome do Desconforto Respiratório Agudo, pneumonia e obstrução de vias aéreas superiores e inferiores, mas também, patologias tais como: doenças neuromusculares, distúrbios do sistema nervoso central, complicações cirúrgicas e trauma. A necessidade de ventilação mecânica, também, pode ser secundária à falência de outros sistemas como cardiovascular, renal e hepático. ${ }^{5}$

Apesar de ter mudado o curso e a mortalidade da insuficiência respiratória aguda, a ventilação mecânica está associada a inúmeras complicações. A intubação orotraqueal prejudica os mecanismos naturais de defesa das vias aéreas, causando inflamação, prejuízo da função ciliar e colonização por bactérias patogênicas. Todos esses fatores aumentam o risco de desenvolvimento de infecção nosocomial principalmente pneumonia associada à ventilação mecânica e às sinusopatias. A pneumonia associada à ventilação mecânica é a segunda causa mais comum de infecção nosocomial em terapia intensiva pediátrica (mais de $20 \%$ das infecções 
nosocomiais). ${ }^{6,7,8,9}$ Ela está associada a maior tempo de internação e aumento de mortalidade. A etiologia parece ser semelhante aos adultos com predominância de bacilos Gram negativos secundários a aspiração de material colonizado da orofaringe ou estômago. A sinusite é mais comum em pacientes com cânula nasotraqueal, já que esta bloqueia a drenagem de secreções dos seios paranasais. Além das complicações infecciosas pode ocorrer lesão pulmonar induzida pela ventilação ${ }^{10,11}$ e, também, complicações na via aérea superior no ponto de contato entre a cânula endotraqueal e a mucosa. Essa interação pode resultar em ulceração, edema e hemorragia da mucosa com potencial para desenvolvimento de estenose subglótica. ${ }^{12}$ Ainda, a intubação e aspiração da via aérea causam grande desconforto para os pacientes, e consequente aumento da necessidade de sedação e analgesia. Portanto, é imperativo que o suporte ventilatório seja descontinuado o mais precocemente possível.

\subsection{Desmame da Ventilação Mecânica}

Qualquer que seja a indicação, iniciar a ventilação mecânica é tarefa relativamente fácil. No entanto, permanece um grande desafio na prática clínica, predizer quando um paciente está apto a sustentar sua respiração de forma independente e confiável e, portanto, garantir que o suporte ventilatório seja removido com sucesso..$^{5,13,14,15}$ Essa dificuldade fica 
evidente quando observamos índices de falha de extubação que variam em torno de $16 \%$ a $19 \%$ na faixa etária pediátrica. ${ }^{16,17,18,19,20,21}$

A falha de extubação definida como a necessidade de reintubação e reinstituição da ventilação mecânica, no período de 48 a 72 horas após a remoção da cânula traqueal, ${ }^{16,17,18,19,20,21,22}$ está associada a um pior prognóstico na faixa etária pediátrica, já que foi demonstrado um aumento de mortalidade, tanto dentro da unidade de terapia intensiva como hospitalar nesse grupo de pacientes. ${ }^{23,24}$

Se esse pior prognóstico está relacionado exclusivamente à falha de extubação ou a seleção de pacientes com doenças mais graves ainda não está bem definido, porém, a falha de extubação parece desempenhar um papel importante na evolução do paciente.

No entanto, prolongar tempo de ventilação mecânica significa aumentar o risco de ocorrer alguma das inúmeras complicações associadas à ventilação mecânica. Quando observamos dados do trabalho de Kurachek $^{24}$ em que $62,5 \%$ das 136 crianças que apresentaram extubação acidental não precisaram ser reintubadas, temos um indício de que, muitas vezes prolongamos o tempo de ventilação desnecessariamente.

Extubar precocemente, porém com sucesso, é uma combinação difícil já que os critérios para indicar extubação são muitas vezes subjetivos e pouco definidos. Os critérios mais utilizados em pediatria são: resolução ou melhora da causa que indicou a intubação, controle farmacológico de broncoespasmo, ausência de acidose respiratória, potencial hidrogeniônico $(\mathrm{pH})$ maior que 7,3 , fração inspirada de oxigênio $\left(\mathrm{FiO}_{2}\right)$ menor ou igual a 
$40 \%$, pressão expiratória final positiva (PEEP) menor ou igual a 5 centímetros de água $\left(\mathrm{cmH}_{2} \mathrm{O}\right)$, temperatura corpórea menor que $38,5^{\circ} \mathrm{C}$, sem sedação contínua, correção dos distúrbios eletrolíticos e ausência de bloqueador neuromuscular 24 horas antes do início do desmame. ${ }^{25}$

A procura por critérios mais objetivos para indicar a remoção da ventilação mecânica torna-se então prioridade. Recentemente, vários trabalhos na faixa etária pediátrica têm sido publicados procurando determinar qual seria o melhor preditor de extubação bem sucedida. Vários autores 16,20,21,25 falharam em determinar um único parâmetro que, isoladamente, pudesse prever de forma acurada o sucesso ou falha de extubação. Eles determinaram que a falha da extubação deve-se a uma série de fatores em conjunto tais como: esforço muscular deficiente caracterizado por volume corrente baixo, aumento da demanda dos músculos respiratórios ou diminuição do "drive" respiratório.

Nesse contexto, surge o teste de respiração espontânea como tentativa de identificar os pacientes aptos para a retirada do ventilador. A intenção do teste é reconstituir, da melhor forma possível, enquanto o paciente ainda está intubado, o trabalho respiratório que terá após extubação e monitorizar qualquer evidência de fadiga muscular. Estudo publicado por Farias et al. ${ }^{25}$ demonstrou que o teste com tubo T foi capaz de predizer extubação com sucesso em cerca de $70 \%$ das crianças a ele submetidas. Num trabalho subsequente ${ }^{23}$ o mesmo grupo demonstrou que o teste de respiração espontânea realizado com tubo $\mathrm{T}$ ou com suporte de pressão apresentou resultados semelhantes na identificação desses 
pacientes. Chavez et al. $^{26}$ realizou o teste de respiração espontânea com CPAP de $5 \mathrm{cmH}_{2} \mathrm{O}$ em 70 crianças e extubou todas ao final do teste independente do resultado. $91 \%$ das crianças passaram no teste foram extubadas com uma porcentagem de $7,8 \%$ de falha de extubação. As crianças não passaram no teste e, também, foram extubadas (9\%) apresentando uma taxa de $50 \%$ de falha de extubação. Ainda não está bem definido qual seria a melhor forma de realizar o teste de respiração espontânea, porém, fica evidente pelos estudos descritos, que o teste pode ser realizado na faixa etária pediátrica e é capaz de identificar aquelas que estão aptas a serem extubadas.

Vários estudos em pediatria $27,29,30,31$ procuraram estudar uma forma de reduzir tempo de ventilação mecânica porém, nenhum deles, conseguiu mostrar uma redução significativa. Estudo multicêntrico realizado por Randolph e col. ${ }^{27}$ falhou em demonstrar diferença significante na mediana de tempo de desmame das crianças randomizadas em três grupos (pressão de suporte, volume suporte ou sem protocolo definido). Alguns estudos conseguiram mostrar uma redução no tempo de desmame, porém, sem uma diminuição concomitante no tempo total de ventilação mecânica. ${ }^{15,29,31}$

Em adultos, Ely et al. ${ }^{32}$ demonstrou que a realização do teste de respiração espontânea em pacientes selecionados pode reduzir o tempo de ventilação mecânica, o custo do tratamento e complicações associadas ao tratamento.

Recentemente, alguns autores ${ }^{13,27,28}$ têm sugerido que a associação de avaliações diárias e teste de respiração espontânea poderia ser uma 
forma de reduzir tempo de ventilação mecânica na faixa etária pediátrica. No entanto, essa associação ainda não foi formalmente estudada na criança. Permanece, portanto, a dúvida se a realização de uma avaliação diária para identificar aquelas crianças aptas a iniciar o desmame e a subsequente realização de um teste de respiração espontânea pode reduzir o tempo de ventilação mecânica, em relação ao desmame habitualmente realizado em pediatria, baseado em critérios clínicos e laboratoriais. 
2 ObJetivos 
São, portanto, objetivos desse estudo:

\title{
2.1 Principal
}

\author{
Avaliar os efeitos da aplicação de avaliações diárias e teste de \\ respiração espontânea sobre a duração da ventilação mecânica em \\ crianças.
}

\subsection{Secundários}

Em pacientes pediátricos sob ventilação mecânica, por um período superior a 24 horas, comparar dois grupos e determinar:

- Taxa de falha de extubação através da necessidade de reintubação no período de 48 horas após extubação.

- Necessidade de ventilação mecânica não-invasiva após extubação.

- Ocorrência de complicações associadas à VM: pneumonia associada à ventilação mecânica e extubação acidental.

- Fatores associados à duração da ventilação mecânica. 
3 MÉtodos 
Esse estudo foi aprovado pelos comitês de ética em pesquisa de ambos os hospitais participantes e um termo de consentimento livre e esclarecido foi obtido de todos os parentes ou responsáveis pelas crianças elegíveis para o estudo, antes da randomização. (Anexos 7.1 e 7.2)

Foram consideradas elegíveis, crianças de ambos os sexos que estivessem em ventilação mecânica por um período superior a 24 horas, com idade entre 28 dias de vida e 15 anos, que foram admitidas nas unidades de terapia intensiva pediátrica (UTIp) do Instituto da Criança do Hospital das Clínicas da Universidade de São Paulo e do Hospital Universitário, no período de julho de 2007 à julho de 2009.

Os critérios de exclusão foram: intubação por Obstrução de Vias Aéreas Superiores (OVAS), presença de hérnia diafragmática ou paralisia diafragmática, uso crônico de ventilação mecânica, cardiopatia congênita cianogênica, hipertensão pulmonar primária, doença neuromuscular e traqueostomia.

Uso crônico de ventilação mecânica foi definido para pacientes dependentes de ventilação mecânica invasiva ou não invasiva, antes da admissão nas UTIp, incluindo crianças de outras unidades do mesmo hospital, de outros hospitais ou unidades de tratamento domiciliar (Home Care). 
Os pacientes podiam participar do estudo somente uma vez durante sua internação hospitalar e, somente, a primeira intubação foi considerada para análise.

O estudo foi registrado no "International Standard Randomized Controlled Trial Number Register" (ISRCTN37806223)

\subsection{Desenho do estudo}

O desenho do estudo pode ser visualizado na figura 1. Os pacientes foram alocados de uma forma aleatória em dois grupos: grupo teste e grupo controle. O grupo teste foi submetido a um protocolo de desmame que combinava avaliações diárias e teste de respiração espontânea (TRE).

A avaliação diária (Anexo 7.3) foi realizada, todas as manhãs até a extubação do paciente, por residentes especialmente treinados para esse procedimento. O treinamento foi realizado antes do início do estudo e posteriormente a cada 3 meses. Esses residentes não eram responsáveis pela decisão de extubação. Além disso, os casos novos, nas duas instituições foram avaliados, uma vez por semana, para verificar se os critérios de inclusão e exclusão estavam sendo seguidos e se as avaliações diárias estavam sendo realizadas de forma apropriada. As crianças que passavam na avaliação diária eram submetidas a um TRE. (Anexo 7.7)

No grupo controle foi realizada retirada gradual através de critérios clínicos e laboratoriais segundo a rotina habitual nos dois serviços. Resumidamente, os modos e parâmetros ventilatórios eram determinados 
pelo médico responsável pelo plantão no dia, sem nenhuma influência do protocolo de estudo. Os modos ventilatórios mais utilizados eram PS, ventilação mandatória intermitente sincronizada (SIMV) e pressão controlada. A redução da frequência respiratória $(\mathrm{FR})$ e pressão inspiratória $(\mathrm{PI})$ era realizada através da monitorização da ventilação do paciente incluindo: expansão torácica, volume exalado, gás carbônico exalado $\left(\mathrm{EtCO}_{2}\right)$ ou da pressão arterial de gás carbônico $\left(\mathrm{PaCO}_{2}\right)$. A fração inspirada de oxigênio $\left(\mathrm{FiO}_{2}\right)$ e a PEEP eram reduzidas de acordo com a oxigenação do paciente para manter uma saturação de oxigênio entre 92 e 98\%.

Os dois grupos de estudo foram cuidados pela mesma equipe médica e os membros da equipe não sabiam para que grupo a criança tinha sido randomizada, até o momento em que o teste de respiração espontânea era indicado.

Os ventiladores utilizados no estudo foram: Newport E500 (Newport Medical Instruments, Newport Beach, CA), Servo-i (Siemens, Elema, Solna, Sweden) and Evita 4 (Dräger, Lübeck, Germany).

Para controlar possíveis diferenças entre as condutas médicas nas duas instituições, a randomização foi estratificada por hospital. A randomização foi realizada através de sorteio. Um saco plástico opaco que continha um número variado de envelopes $(6,12$ ou 20) era preparado por uma pessoa não envolvida com o cuidado do paciente, sempre contendo metade dos envelopes direcionados para cada grupo. O mesmo residente responsável pelas avaliações diárias, sorteava no momento da randomização o envelope que continha a informação para qual grupo aquele 
paciente seria direcionado. Assim que terminavam todos os envelopes de um saco plástico, este era substituído por um novo. A randomização era feita numa razão de 1:1.

\subsection{Procedimentos}

Para passar na avaliação diária o paciente deveria preencher os seguintes critérios:

- $\mathrm{FiO}_{2} \leq 50 \%$

- Pressão Expiratória Final Positiva (PEEP) $\leq 8$

- Pressão Inspiratória $(\mathrm{PI}) \leq 25 \mathrm{~cm} \mathrm{H} 2 \mathrm{O}$

- Ausência de novos infiltrados na radiografia torácica (RX)

- Presença de "drive" respiratório

- Ausência de sedação contínua com um nível de consciência adequado

- Sem uso de bloqueador neuromuscular nas últimas 24 horas

- Correção de alterações hidroeletrolíticas (cálcio, magnésio, fósforo e potássio)

- Estabilidade hemodinâmica (permitido doses de dopamina ou dobutamina até $10 \mathrm{mcg} / \mathrm{kg} / \mathrm{min}$ e nitroprussiato de sódio em qualquer dose)

- Hemoglobina $\geq 8 \mathrm{~g} / \mathrm{dL}$ 
Para avaliação do nível de consciência foi utilizada a escala de Comfort-Behavior (anexo 7.4) e foi considerado adequado caso os pacientes apresentassem um escore superior ou igual a 9.

Os pacientes que preenchiam esses critérios foram, então, submetidos a um TRE com PEEP de $5 \mathrm{cmH} 2 \mathrm{O}$ e PS de $10 \mathrm{cmH}_{2} \mathrm{O}$ e mesma $\mathrm{FiO}_{2}$ que já estava em uso por um período de 2 horas. O teste era interrompido a qualquer momento se houvesse algum indício de intolerância.

Os critérios para interrupção do teste incluíam: aumento da Frequência Respiratória (FR) ou Frequência Cardíaca (FC) acima de $20 \%$ dos valores basais (antes do início do TRE); sinais de aumento do trabalho respiratório (uso de musculatura acessória ou respiração paradoxal); alteração no nível de consciência; Pressão Arterial (PA) abaixo do percentil 5 para idade; Saturação arterial de Oxigênio $\left(\mathrm{SaO}_{2}\right)$ menor $90 \%$ ou $\mathrm{PaCO}_{2}$ maior que $50 \mathrm{mmHg}$ ou um aumento superior $10 \mathrm{mmHg}$ por hora em pacientes com doença pulmonar crônica. Foram colhidas gasometrias arteriais antes do TRE e após 1 hora de teste. Os pacientes que completavam o teste sem nenhum dos critérios de falha eram extubados. Se detectado qualquer sinal de intolerância o teste era interrompido e a VM era reiniciada nos parâmetros prévios. O teste era então novamente realizado após 24 horas. (Figura 1) 


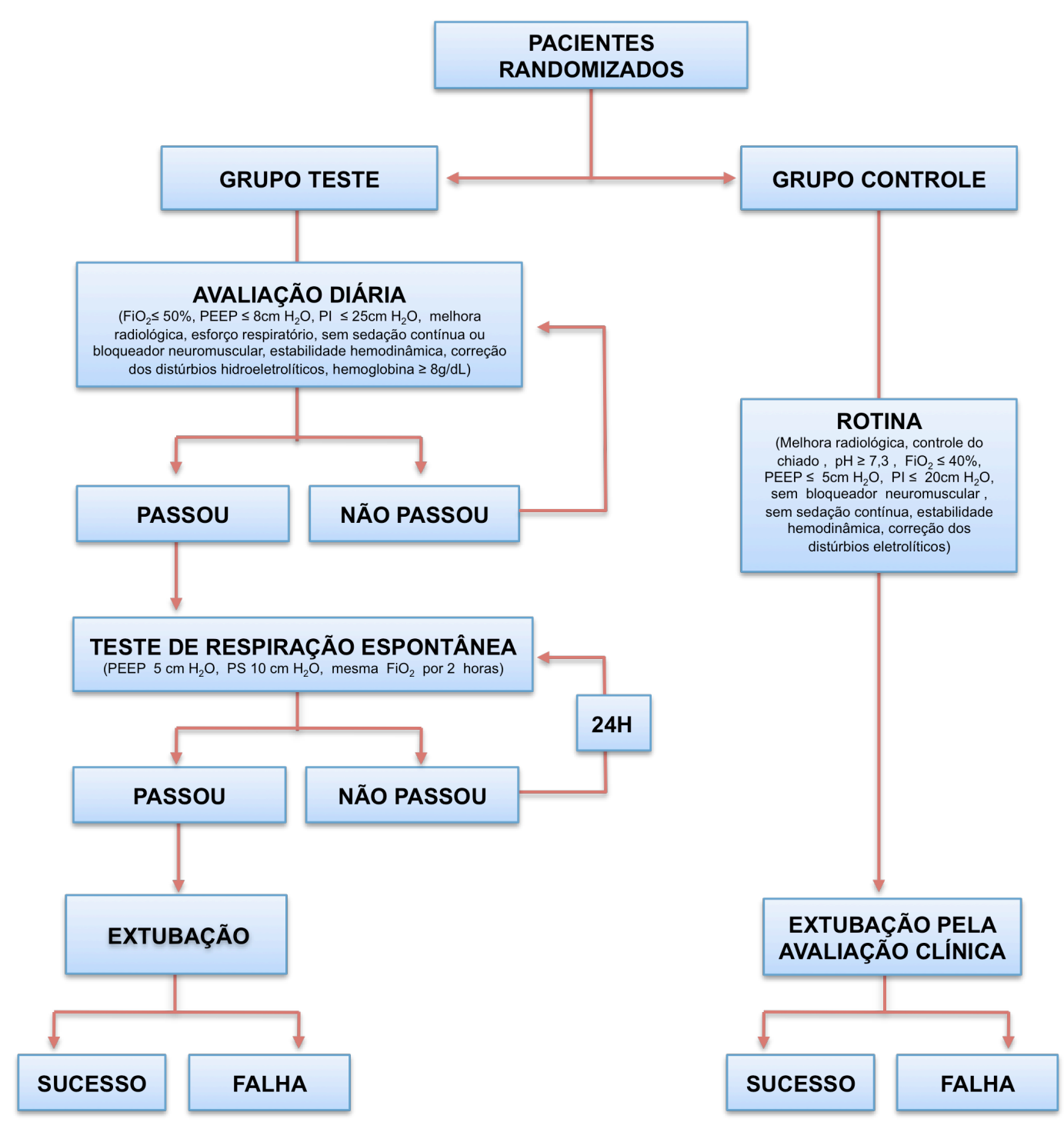

$\mathrm{FiO}_{2}$ : Fração Inspirada de Oxigênio; PEEP: Pressão Expiratória Final Positiva; PI: Pressão Inspiratória; PS: Pressão de Suporte; pH: potencial Hidrogeniônico; $\mathrm{H}$ : Horas; $\mathrm{cm} \mathrm{H}_{2} \mathrm{O}$ : centímetros de água

\section{Figura 1: Desenho do estudo}




\subsection{Desmame e extubação}

No paciente do grupo teste o desmame foi realizado da mesma forma que no grupo controle por critérios clínicos e gasométricos até que o paciente preenchesse os critérios da avaliação diária, quando então, era indicado o TRE. Os critérios, nas duas instituições, para indicar extubação foram:

- Resolução ou controle da causa que levou a intubação

- Controle farmacológico do broncoespasmo

- Ausência de acidose respiratória $(\mathrm{pH}>7,3)$

- $\mathrm{FiO}_{2} \leq 40 \% ; \mathrm{PEEP} \leq 5 \mathrm{~cm} \mathrm{H} \mathrm{O}_{2} ; \mathrm{PI} \leq 20 \mathrm{~cm} \mathrm{H}_{2} \mathrm{O}$

- Sem necessidade de sedação contínua

- Estabilidade hemodinâmica

- Correção de distúrbios hidroeletrolíticos

- Sem necessidade de bloqueador neuromuscular nas últimas 24 horas

Após extubação o paciente era monitorizado por 48 horas. Falha de extubação era definida como necessidade de reintubação nesse período. Reintubação era indicada para aqueles pacientes que tinham 2 ou mais dos seguintes critérios clínicos: aumento da frequência respiratória maior que $40 \%$ da FR normal para idade (Anexo 7.5); apneia por período superior a 20 segundos; retrações subdiafragmáticas ou intercostais; cianose ou por critérios gasométricos: $\mathrm{PaO}_{2} / \mathrm{FiO}_{2}<200$ ou $\mathrm{PaCO}_{2}>50 \mathrm{~mm} \mathrm{Hg} \mathrm{c} \mathrm{pH}<7,35$. Ventilação não invasiva era indicada nos casos de desconforto respiratório, 
hipoxemia (saturação < 90\%), diminuição do nível de consciência levando a um esforço respiratório inadequado, OVAS e insuficiência cardíaca. Se os pacientes em VNI, com dois níveis de pressão, eram incapazes de manter uma $\mathrm{PaO}_{2} \geq 80$ mm Hg com uma $\mathrm{FiO}_{2} \leq 60 \%$ era indicada a reintubação.

\subsection{Cálculo Amostral}

Para o cálculo do tamanho amostral, baseado na experiência prévia nas duas UTIp participantes, a inclusão de 133 pacientes por grupo dariam ao estudo um poder de $80 \%$, detectando um aumento na proporção de pacientes extubados com sucesso, no quarto dia de ventilação, de $35 \%$ no grupo controle para $50 \%$ no grupo teste. Consideramos uma confiança da amostra de $95 \%$. O estudo foi analisado por intenção de tratamento.

\subsection{Análise estatística}

As seguintes variáveis foram coletadas e analisadas em todos os pacientes: idade, sexo, diagnóstico primário, presença de doenças de base, indicação de ventilação mecânica, escores de risco de mortalidade pediátricos $\left(\mathrm{PRISM}^{41}\right.$ e PIM $\left.2^{42}\right)$, complicações respiratórias, duração da ventilação mecânica, parâmetros ventilatórios, uso de drogas vasoativas e sedativas, falha de extubação e uso de ventilação não invasiva. 
As variáveis quantitativas foram testadas para avaliar a normalidade de distribuição de probabilidade dos dados com uso de testes KolmogorovSmirnov (Conover, 1980).

A comparação das variáveis quantitativas entre os grupos foi realizada com uso de testes Mann-Whitney (Conover, 1980) quando havia falta de normalidade de distribuição dos parâmetros, e testes t-Student quando houve normalidade de distribuição dos parâmetros.

Os testes qui-quadrado ou teste exato de Fisher (Bussab e Morettin, 1987) foram aplicados para verificar a existência de associação das variáveis qualitativas entre os grupos.

O tempo para extubação foi estimado por meio de curvas de sobrevida obtidas pelo método de Kaplan-Meier e as comparações entre as curvas foram realizadas usando o teste log-rank. Tempo de ventilação foi definido como tempo entre o início da ventilação mecânica e a primeira extubação. Somente os pacientes extubados foram considerados na análise da duração da ventilação mecânica e dos desfechos secundários; aqueles que faleceram sem nunca terem sido desmamados e extubados foram excluídos da análise desses desfechos. Um período de 30 dias de seguimento foi considerado na análise. Os pacientes em VM, por um período superior a 30 dias, foram considerados como falha de desmame e foram censorados na análise.

O modelo de regressão de Cox foi usado na análise múltipla para as variáveis com $p<0,20$ na análise univariada. 
4 Resultados 


\subsection{Características gerais dos pacientes}

Foram selecionados um total de 776 pacientes, mas 464 não preenchiam os critérios de inclusão e exclusão (Figura 2). Dos 312 pacientes randomizados 18 não eram elegíveis: 11 pacientes ficaram em VM por um período inferior a 24h; 3 apresentavam doença neuromuscular; 2 tinham OVAS; 1 paciente tinha mais de 15 anos; 1 paciente foi excluído por ser sua segunda intubação. Dessas violações de protocolo 8 pertenciam ao grupo teste e 10 pertenciam ao grupo controle (Figura 2). Esses pacientes foram excluídos da análise.

Dos 294 pacientes elegíveis randomizados, 155 pertenciam ao grupo teste e 139 pertenciam ao grupo controle. 


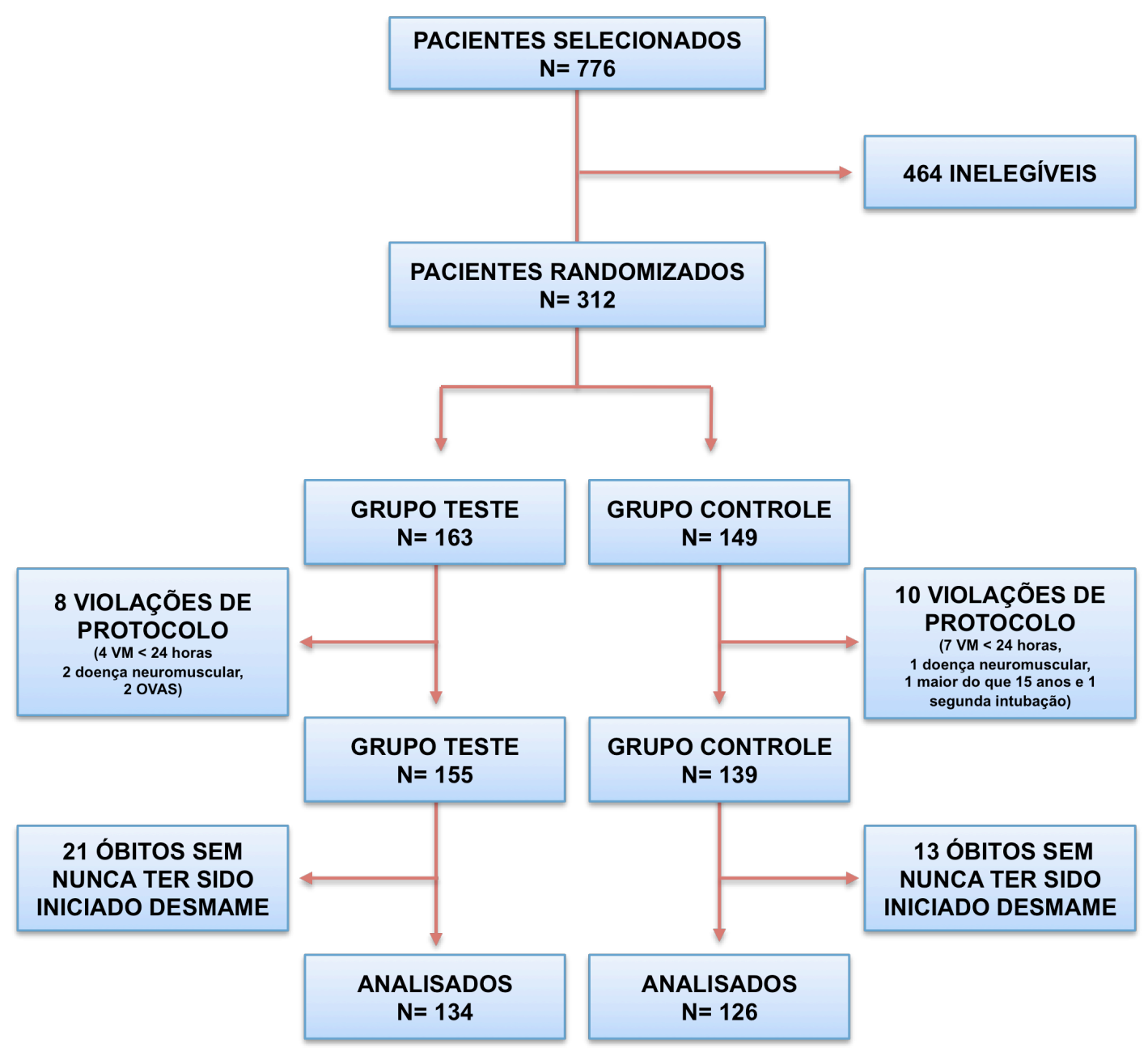

VM: Ventilação Mecânica; OVAS: Obstrução de Vias Aéreas Superiores

\section{Figura 2: Fluxo de pacientes}

Os pacientes tinham uma idade mediana de 11 meses e $54,8 \%$ eram do sexo masculino. A Tabela 1 resume as características demográficas e clínicas basais dos pacientes randomizados. Os grupos não diferem em idade, sexo e nos escores de risco de mortalidade (PRISM e PIM 2). O diagnóstico primário também foi semelhante nos dois grupos com exceção 
da presença de tumores malignos que foi mais incidente no grupo teste. Também não foram encontradas diferenças significantes entre os grupos nas principais indicações de ventilação mecânica. Finalmente, não foi encontrada nenhuma diferença entre os grupos quanto à taxa de mortalidade observada durante o estudo (14,8\% no grupo teste e $10,8 \%$ no grupo controle; $p=0,302)$.

Tabela 1 - Características clínicas e demográficas dos pacientes randomizados de acordo com o grupo de estudo.

\begin{tabular}{|c|c|c|c|}
\hline & Grupo teste $(n=155)$ & Grupo controle $(n=139)$ & $\mathbf{P}$ \\
\hline Idade (anos) ${ }^{*}$ & $0,922[0,303 ; 3,483]$ & $0,933[0,344 ; 3,399]$ & 0,918 \\
\hline PRISM* & $0,050[0,025 ; 0,118]$ & $0,051[0,128 ; 0,275]$ & 0,756 \\
\hline PIM2* & $0,098[0,040 ; 0,244]$ & $0,080[0,036 ; 0,177]$ & 0,148 \\
\hline Sexo masculino** & $86(55,5 \%)$ & $75(54 \%)$ & 0,793 \\
\hline Imunodeficiência** & $7(4,5 \%)$ & $6(4,3 \%)$ & 0,934 \\
\hline Cardiopatia congênita** & $9(5,8 \%)$ & $8(5,8 \%)$ & 0,985 \\
\hline Doenças genéticas ${ }^{* *}$ & $7(4,5 \%)$ & $7(5 \%)$ & 0,834 \\
\hline Tumores malignos** & $12(7,7 \%)$ & $3(2,2 \%)$ & 0,030 \\
\hline Encefalopatias ${ }^{* *}$ & $17(11 \%)$ & $9(6,5 \%)$ & 0,176 \\
\hline Doença hepática** & $13(8,4 \%)$ & $20(14,4 \%)$ & 0,104 \\
\hline Doença pulmonar crônica** & $20(12,9 \%)$ & $19(13,7 \%)$ & 0,847 \\
\hline Leucemia** $^{* *}$ & $5(3,2 \%)$ & $6(4,3 \%)$ & 0,623 \\
\hline \multicolumn{4}{|l|}{$\begin{array}{l}\text { Principais indicações da } \\
\text { ventilação mecânica }\end{array}$} \\
\hline $\mathrm{ICC}^{* *}$ & $4(2,6 \%)$ & $9(6,5 \%)$ & 0,105 \\
\hline Chiado** & $34(21,9 \%)$ & $31(22,3 \%)$ & 0,940 \\
\hline Choque séptico** & $35(22,6 \%)$ & $20(14,4 \%)$ & 0,072 \\
\hline Coma** & $24(15,5 \%)$ & $14(10,1 \%)$ & 0,167 \\
\hline Pneumonia** & $60(38,7 \%)$ & $60(43,2 \%)$ & 0,576 \\
\hline Bronquiolite** & $27(17,4 \%)$ & $34(24,5 \%)$ & 0,200 \\
\hline SDRA $^{* *}$ & $13(8,4 \%)$ & $8(5,7 \%)$ & 0,382 \\
\hline
\end{tabular}

ICC: Insuficiência Cardíaca Congestiva; SDRA: Síndrome do Desconforto Respiratório Agudo; PRISM: Risco de Mortalidade Pediátrica; PIM2: Índice de Mortalidade Pediátrica

Valores são: medianas [P25 e P75] ou números (percentis)

* Teste de Mann-Whitney; ${ }^{* *}$ Teste Qui quadrado 
Como os objetivos primários e secundários do estudo eram baseados nos pacientes que haviam sido extubados ou, ainda, estavam em ventilação mecânica durante um período de 30 dias de seguimento, 34 pacientes que faleceram em ventilação mecânica sem nunca terem sido submetidos à desmame e extubação foram excluídos das análises subsequentes. Desses pacientes 21 pertenciam ao grupo teste e 13 ao grupo controle sendo, portanto, considerados para as análises dos objetivos primários e secundários 134 pacientes no grupo teste e 126 pacientes no grupo controle (Figura 2). No grupo teste, 5 pacientes foram extubados sem a realização de um teste de respiração espontânea (3 pacientes por decisão médica, 1 por agitação psicomotora durante o teste e 1 por extubação acidental). No grupo controle 17 pacientes foram extubados após a realização de um teste de respiração espontânea sendo que a maior parte dos casos ocorreu nos últimos 2 meses de estudo. É importante ressaltar que todos os pacientes foram analisados nos grupos para o qual foram alocados seguindo os princípios de intenção de tratamento. A Tabela 2 resume as características basais dos pacientes que foram considerados para as análises dos objetivos primários e secundários.

Nos dois grupos a principal causa de admissão foi clínica e não cirúrgica (92,9\% no grupo controle e $96,3 \%$ no grupo teste; $p=0,223)$. As principais indicações de ventilação mecânica foram: pneumonia (42,3\%); broncoespasmo (24,6\%); bronquiolite (23\%); choque séptico $(16,9 \%)$ e coma (12,3\%). Síndrome do Desconforto Respiratório Agudo (SDRA) foi presente em apenas $5 \%$ dos pacientes. Não encontramos diferenças 
significativas entre os grupos quanto às principais indicações de ventilação mecânica (Tabela 2).

Tabela 2 - Características clínicas e demográficas dos pacientes analisados de acordo com o grupo de estudo

\begin{tabular}{|c|c|c|c|}
\hline & Grupo teste $(n=134)$ & Grupo controle $(n=126)$ & $\mathbf{P}$ \\
\hline Idade (anos)* & $1,160[0,371 ; 3,506]$ & $0,712[0,254 ; 2,172]$ & 0,070 \\
\hline PRISM* & $0,042[0,023 ; 0,097]$ & $0,051[0,028 ; 0,128]$ & 0,419 \\
\hline PIM2* & $0,091[0,033 ; 0,188]$ & $0,081[0,036 ; 0,180]$ & 0,598 \\
\hline Sexo masculino** & $71(53,0 \%)$ & $72(57,1 \%)$ & 0,501 \\
\hline Imunodeficiência** & $4(3,0 \%)$ & $3(2,4 \%)$ & 0,764 \\
\hline Cardiopatia congênita** & $6(4,5 \%)$ & $8(6,3 \%)$ & 0,504 \\
\hline Doenças genéticas ${ }^{* *}$ & $7(5,2 \%)$ & $6(4,8 \%)$ & 0,864 \\
\hline Tumores malignos** & $8(6,0 \%)$ & $3(2,4 \%)$ & 0,151 \\
\hline Neuropatias ${ }^{* *}$ & $15(11,2 \%)$ & $9(7,1 \%)$ & 0,259 \\
\hline Doença hepática** & $7(5,2 \%)$ & $15(11,9 \%)$ & 0,053 \\
\hline Doença pulmonar crônica** & $20(14,9 \%)$ & $17(13,5 \%)$ & 0,741 \\
\hline Leucemia $^{* *}$ & $4(3,0 \%)$ & $5(4,0 \%)$ & 0,665 \\
\hline \multicolumn{4}{|l|}{$\begin{array}{l}\text { Principais indicações da } \\
\text { ventilação mecânica }\end{array}$} \\
\hline ICC** & $3(2,2 \%)$ & $6(4,8 \%)$ & 0,266 \\
\hline Chiado** & $34(25,4 \%)$ & $30(23,8 \%)$ & 0,770 \\
\hline Choque séptico** & $26(19,4 \%)$ & $18(14,3 \%)$ & 0,271 \\
\hline Coma** & $19(14,2 \%)$ & $13(10,3 \%)$ & 0,344 \\
\hline Pneumonia** & $55(41,0 \%)$ & $55(43,7 \%)$ & 0,671 \\
\hline Bronquiolite $^{* *}$ & $28(20,9 \%)$ & $32(25,4 \%)$ & 0,389 \\
\hline SDRA $^{* *}$ & $7(5,2 \%)$ & $6(4,8 \%)$ & 0,864 \\
\hline
\end{tabular}

ICC: Insuficiência Cardíaca Congestiva; SDRA: Síndrome do Desconforto Respiratório Agudo; PRISM: Risco de mortalidade pediátrica; PIM2: Índice de Mortalidade Pediátrica

Valores são: medianas [P25 e P75] ou números (percentis)

*Teste de t-Student; ** Teste Qui quadrado 
Foi realizada a comparação do estado nutricional nos dois grupos através do cálculo do Escore z de peso para idade nas crianças menores de 10 anos e Escore z do índice de massa corpórea (IMC) para crianças acima de 10 anos. Apenas em 13 crianças ( 9 crianças do grupo teste e 4 crianças do grupo controle) não foi possível achar o dado de estatura para cálculo do IMC. Para tal comparação foram utilizadas as curvas propostas pela Organização Mundial de Saúde (OMS) publicadas em 2006 e 2007, bem como, as classificações propostas nessa publicação. Foi considerado magreza acentuada crianças com Escore z menor que -3, magreza um Escore $z$ entre -3 e -2 e eutróficas aquelas com um Escore $z$ entre -2 e +1 . Em crianças até 5 anos um Escore $z$ de $+1 \mathrm{a}+2$ foi considerado risco de sobrepeso, de +2 a +3 sobrepeso e maior que +3 obesidade. Para crianças acima de 5 anos um Escore $z$ de $+1 \mathrm{a}+2$ foi considerado sobrepeso, de $+2 \mathrm{a}+3$ obesidade $\mathrm{e}$ maior que +3 obesidade grave. Não foi identificada nenhuma diferença estatisticamente significativa entre os grupos quanto ao nível de classificação nutricional ( $p=0,325$; teste de Mann-Whitney) (Tabela 3).

Tabela 3 - Comparação dos grupos quanto ao estado nutricional

\begin{tabular}{lcccccc}
\hline \multirow{2}{*}{ Escore Z } & \multicolumn{2}{c}{ Grupo controle } & \multicolumn{2}{c}{ Grupo teste } & \multicolumn{2}{c}{ Total } \\
& $\mathbf{N}$ & $\mathbf{\%}$ & $\mathbf{N}$ & $\mathbf{\%}$ & $\mathbf{N}$ & $\%$ \\
\hline Magreza acentuada & 14 & 11,5 & 7 & 5,6 & 21 & 8,5 \\
\hline Magreza & 16 & 13,1 & 13 & 10,4 & 29 & 11,7 \\
Eutrofia & 71 & 58,2 & 84 & 67,2 & 155 & 62,8 \\
\hline Risco de sobrepeso & 9 & 7,4 & 16 & 12,8 & 25 & 10,1 \\
Sobrepeso & 8 & 6,6 & 4 & 3,2 & 12 & 4,9 \\
\hline Obesidade & 4 & 3,3 & 0 & 0,0 & 4 & 1,6 \\
Obesidade grave & 0 & 0,0 & 1 & 0,8 & 1 & 0,4 \\
\hline
\end{tabular}

$\mathrm{N}$ : número de pacientes; \%: percentual de pacientes 
Comparando a ocorrência de disfunções orgânicas durante o período de ventilação mecânica nos dois grupos, a frequência de choque foi maior no grupo teste do que no grupo controle $(12,7 \%$ vs. $5,6 \% ; p=0,047)$. Todas as outras situações avaliadas tiveram frequências semelhantes nos dois grupos: SDRA $(p=0,585)$; sepse $(p=0,218)$; insuficiência renal aguda $(p=0,190)$ e coagulopatia $(p=0,806)$.

Os dois grupos também eram semelhantes quantos aos modos ventilatórios utilizados. A maior parte dos pacientes foram ventilados em modalidades controladas à pressão. Ventilação pressão controlada ou Ventilação Mandatória Intermitente Sincronizada (SIMV) com Pressão de Suporte (PS) foram as modalidades mais utilizadas nas duas UTIp, porém, 21 crianças foram ventiladas no modo Volume Controlado Pressão Regulada (PRVC) sendo 12 crianças no grupo teste e 9 crianças no grupo controle. O modo volume controlado não foi utilizado em nenhum dos casos do estudo. Para finalizar, os dois grupos eram bastante semelhantes quanto ao uso de sedativos sendo os mais utilizados os benzodiazepínicos e opióides e quanto ao uso de drogas vasoativas como dobutamina, dopamina, noradrenalina, milrinone e adrenalina, não havendo significância estatística ( $p>0,05)$.

\subsection{Duração da ventilação mecânica}

A realização de uma análise de Kaplan-Meier do tempo necessário para extubação mostrou que a duração da ventilação mecânica teve uma 
mediana de 3,5 dias no grupo teste (95\% IC: $2,95-4,02)$ e 4,7 dias no grupo controle (95\% IC: 4,08-5,34) (Figura 3). Portanto, num período de 30 dias de seguimento, houve uma redução estatisticamente significante de $30 \%$ no risco da criança permanecer em ventilação mecânica, se ela fosse do grupo teste em comparação ao grupo controle. (Risco Relativo= 0,7; 95\% IC: $0,53-0,93 ; p=0,013)$.

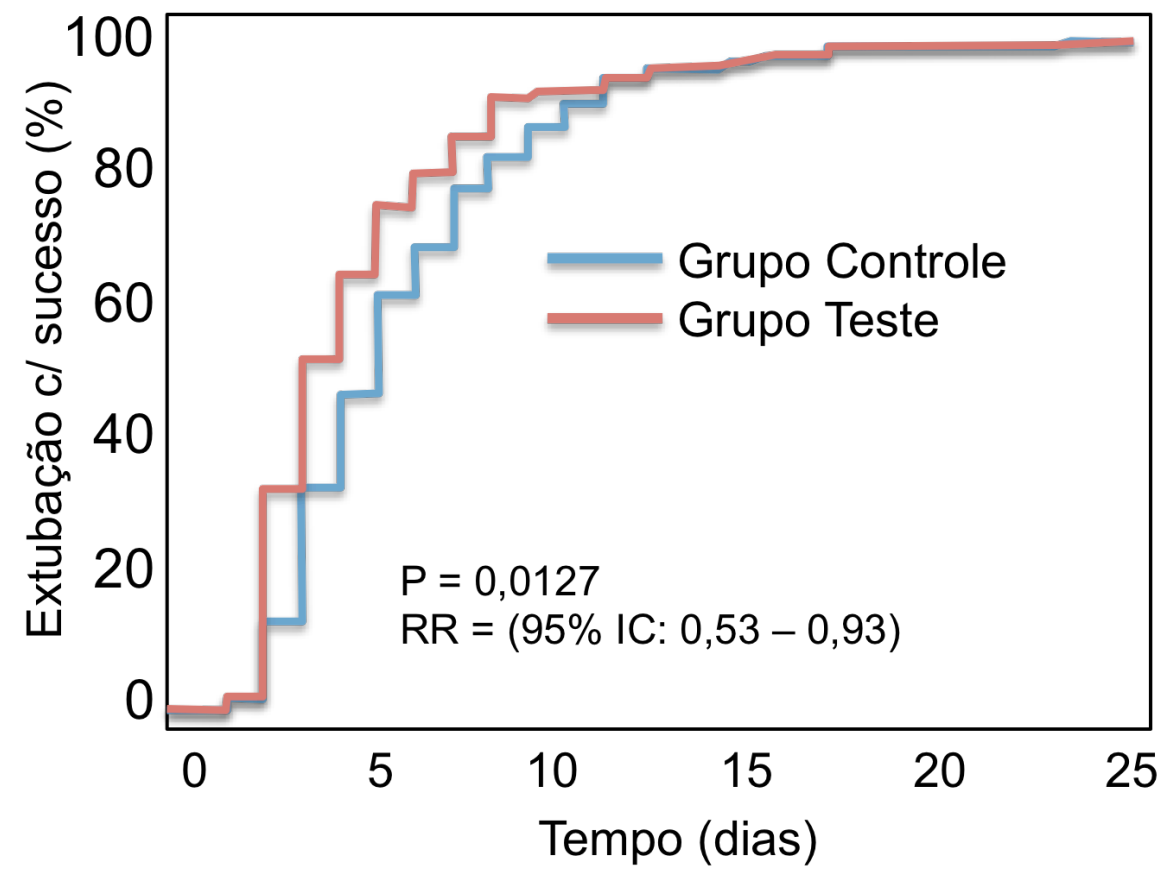

Figura 3: Comparação do tempo p/ extubação entre os grupos

$\mathrm{Na}$ análise univariada os seguintes fatores foram identificados como associados de forma significativa a duração da ventilação mecânica: Pertencer ao grupo teste $(p=0,004) ; \operatorname{PRISM}(p=0,042) ; \operatorname{PIM} 2(p=0,024)$; ser portador de doença hepática $(p=0,024)$ ter SDRA como causa de indicação da VM $(p=0,003)$; apresentar barotrauma $(p=0,006)$ e desenvolver 
SDRA $(p=0,002)$ ou pneumonia associada a ventilação $(p<0,001)$ durante o período de VM. Além desses fatores, foram incluídos na análise múltipla por possuírem um $\mathrm{p}<0,2$ as seguintes variáveis: ser portador de síndrome genética $(p=0,085)$; pneumonia como indicação de ventilação mecânica $(p=0,164)$; desenvolver choque $(p=0,077)$; desenvolver insuficiência renal aguda $(p=0,067)$ e apresentar extubação acidental $(p=0,129)$.

$\mathrm{Na}$ análise múltipla, ajustada por covariantes, pertencer ao grupo teste foi identificado como o único fator associado a um menor tempo de ventilação mecânica $(p<0,001)$, a presença de SDRA como motivo de início da ventilação mecânica $(p<0,001)$ e o desenvolvimento durante a ventilação mecânica de: insuficiência renal aguda $(p=0,018)$; pneumonia associada à ventilação $(p<0,001)$ e SDRA $(p=0,001)$ foram identificados como fatores relacionados a um maior tempo de ventilação (Tabela 4).

Tabela 4 - Análise multivariada dos fatores preditivos do tempo para extubação

\begin{tabular}{lccc}
\hline Variável & RR & [95\%IC] & P \\
\hline Grupo (teste) & 0,60 & {$[0,47 ; 0,78]$} & $<0,001$ \\
SDRA (como indicação de VM) & 3,86 & {$[2,13 ; 6,99]$} & $<0,001$ \\
Presença de IRA & 1,99 & {$[1,12 ; 3,52]$} & 0,018 \\
Pneumonia associada ao ventilador & 3,32 & {$[2,02 ; 5,45]$} & $<0,001$ \\
SDRA (como complicação) & 3,73 & {$[1,77 ; 7,86]$} & 0,001 \\
\hline
\end{tabular}

SDRA: Síndrome do Desconforto Respiratório Agudo; IRA: Insuficiência Renal Aguda;

IC: Intervalo de Confiança; PIM: Índice de Mortalidade Pediátrica; RR: Risco Relativo. 


\subsection{Desfechos secundários}

Essa redução significativa no tempo de ventilação mecânica encontrada no grupo teste não foi associada a um aumento nas taxas de falha de extubação e necessidade de reintubação. Como pode ser observado na tabela 5 , os dois grupos tiveram taxas semelhantes de reintubação, pneumonia associada à ventilação mecânica e extubação acidental. Apesar de ter sido observado uma menor necessidade de VNI no grupo teste em relação ao grupo controle essa diferença não foi significante $(p=0,088)$.

Tabela 5 - Complicações respiratórias nos dois grupos

\begin{tabular}{lccc}
\hline Variável & $\begin{array}{c}\text { Grupo teste } \\
(\mathbf{N = 1 3 4 )}\end{array}$ & $\begin{array}{c}\text { Grupo controle } \\
(\mathbf{N}=126)\end{array}$ & $\mathbf{p}$ \\
\hline $\begin{array}{l}\text { Necessidade de ventilação mecânica } \\
\text { não-invasiva* }\end{array}$ & $29(21,6 \%)$ & $39(31,0 \%)$ & 0,088 \\
\hline Reintubação* & $15(11,2 \%)$ & $18(14,3 \%)$ & 0,454 \\
\hline Pneumonia associada ao ventilador* & $9(6,7 \%)$ & $12(9,5 \%)$ & 0,406 \\
\hline Extubação acidental* & $3(2,2 \%)$ & $8(6,3 \%)$ & 0,100 \\
\hline
\end{tabular}

*Teste do Qui quadrado

É importante ressaltar, que numa análise comparativa dos parâmetros ventilatórios imediatamente antes da extubação não foi encontrada uma diferença significante entre os dois grupos quanto a $\mathrm{FiO}_{2}(p=0,40)$; PEEP $(p=0,35) ; \mathrm{PI}(p=0,71)$ e VC $(p=0,26)$ como pode ser observado na tabela 6. 
Tabela 6 - Comparação dos parâmetros ventilatórios antes da extubação nos dois grupos

\begin{tabular}{|c|c|c|c|}
\hline Parâmetro & Grupo teste & Grupo controle & $\mathbf{P}$ \\
\hline \multicolumn{4}{|l|}{$\mathrm{FiO}_{2}^{*}$} \\
\hline Média $\pm D P$ & $0,33 \pm 0,06$ & $0,34 \pm 0,08$ & \multirow{2}{*}{0,40} \\
\hline Mediana & 0,3 & 0,3 & \\
\hline \multicolumn{4}{|l|}{$\mathrm{PI}\left(\mathrm{cm} \mathrm{H}_{2} \mathrm{O}\right)^{*}$} \\
\hline Média $\pm D P$ & $18,67 \pm 2,83$ & $18,36 \pm 2,44$ & \multirow{2}{*}{0,71} \\
\hline Mediana & 18,0 & 18,0 & \\
\hline \multicolumn{4}{|l|}{ PEEP $\left(\mathrm{cm} \mathrm{H}_{2} \mathrm{O}\right)^{*}$} \\
\hline Média $\pm D P$ & $6,38 \pm 0,93$ & $6,24 \pm 0,66$ & \multirow{2}{*}{0,35} \\
\hline Mediana & 6,0 & 6,0 & \\
\hline \multicolumn{4}{|l|}{ VC $(\mathrm{mL} / \mathrm{kg})^{*}$} \\
\hline Média $\pm D P$ & $7,36 \pm 1,94$ & $6,91 \pm 1,40$ & \multirow{2}{*}{0,26} \\
\hline Mediana & 7,0 & 7,0 & \\
\hline
\end{tabular}

$\mathrm{FiO}_{2}$ : fração inspirada de oxigênio; PI: Pressão Inspiratória; PEEP: Pressão Expiratória Final Positiva; VC: Volume Corrente; DP: Desvio Padrão.

* Teste de Mann-Whitney.

\subsection{Análises exploratórias}

Vinte e uma crianças no grupo teste passaram na avaliação diária, mas falharam no primeiro teste de respiração espontânea. Desses pacientes, 20 passaram num segundo teste de respiração espontânea e, apenas em 1 foi necessário a realização de um terceiro teste. Quando foi avaliado os parâmetros ventilatórios nesse grupo de pacientes a $\mathrm{FiO}_{2}$ mediana foi de 0,3; a mediana do PEEP foi de $6 \mathrm{~cm} \mathrm{H}_{2} \mathrm{O}$ e a mediana da PI foi de $20 \mathrm{~cm} \mathrm{H} \mathrm{H}_{2} \mathrm{O}$. Não houve diferença significativa entre os parâmetros nesse grupo de pacientes em relação ao restante do grupo e não houve 
diferença significante quando comparados os parâmetros do primeiro e os demais testes de respiração espontânea.

Houve 22 casos de desvio de protocolo como foi especificado na descrição das características gerais dos pacientes. Nenhum dos 5 pacientes do grupo teste, que foram extubados sem completar um teste de respiração espontânea, falharam a extubação e necessitaram de reintubação. Dos 17 pacientes do grupo controle que foram extubados após a realização de um teste de respiração espontânea, apenas 1 falhou a extubação e precisou ser reintubado $(5,9 \%)$. Os parâmetros ventilatórios desses 22 pacientes eram semelhantes aos parâmetros do restante da população no momento da extubação $\left(\mathrm{FiO}_{2} \mathrm{p}=0,675 ;\right.$ PEEP $\mathrm{p}=0,855$ e $\left.\mathrm{PI} p=0,895\right)$. Além disso, a análise da duração da ventilação mecânica excluindo esses 22 pacientes que violaram o protocolo confirmou os resultados do estudo.

Numa análise exploratória foram encontrados 20 pacientes que foram submetidos ao TRE com um PEEP de $8 \mathrm{~cm} \mathrm{H} \mathrm{H}_{2} \mathrm{O}$. Entre esses pacientes apenas 2 necessitaram de um segundo TRE e dos 18 pacientes que foram extubados após o primeiro TRE, apenas 2 precisaram ser reintubados $(11,1 \%)$. Nenhum dos pacientes que foram submetidos a um TRE com PEEP de $8 \mathrm{~cm} \mathrm{H}_{2} \mathrm{O}$ morreram durante o estudo. 
5 Discussão 
Apesar de termos, atualmente, um melhor entendimento de como diminuir lesão pulmonar induzida pela ventilação mecânica, ${ }^{33,34,35}$ o tempo em ventilação continua tendo um papel significante. Quanto mais tempo o paciente fica exposto à ventilação, maior o risco de apresentar injuria pulmonar. $^{10,11}$

No entanto, ainda permanece um grande desafio, na faixa etária pediátrica, reduzir duração da ventilação mecânica extubando as crianças o mais precocemente possível. ${ }^{13}$ Portanto, é muito importante estabelecer estratégias que podem predizer se o paciente está apto ou não a ser extubado com sucesso. No presente estudo (Anexo 7.6), conseguimos demonstrar que a introdução de um protocolo de desmame, que combina uma avaliação diária e a realização do TRE para avaliar se a criança está apta a ser extubada, pode reduzir o tempo de ventilação mecânica. Foi observada uma redução na mediana do tempo de VM em 1 dia no grupo intervenção quando comparado ao grupo controle.

Estudos em adultos mostraram que os pacientes nem sempre precisam de um desmame gradual. Quando é implementada uma avaliação diária e um TRE, cerca de $75 \%$ dos adultos estão prontos para serem extubados. $^{32,36}$ Estudos em crianças demonstraram resultados semelhantes. ${ }^{23,25}$ Porém, enquanto os estudos em adultos conseguiram reduzir tempo de ventilação, ${ }^{32,37}$ em pediatria não se sabe se protocolos 
semelhantes poderiam trazer algum benefício. Em um estudo publicado por Randolph e colaboradores ${ }^{27}$ a introdução de um protocolo não demonstrou diferença significativa entre os grupos quanto ao tempo de desmame. Os autores compararam 3 grupos: um grupo com desmame na modalidade pressão de suporte, outro na modalidade volume suporte e o terceiro grupo foi desmamado sem nenhum protocolo específico. A mediana do tempo de desmame nos 3 grupos foi semelhante e a conclusão do estudo foi que o período de desmame é relativamente curto na faixa etária pediátrica e que a introdução de protocolos não devem alterar esse já curto período. No entanto, vale a pena ressaltar que cerca de um terço das crianças elegíveis para o protocolo de desmame passaram num teste de respiração espontânea e foram extubadas antes da randomização para um dos 3 grupos, sendo que a maioria permaneceu extubada por um período superior a 24 horas. Permanece, portanto, a dúvida se um protocolo de desmame poderia indicar mais precocemente quando um paciente está apto à extubação. No nosso estudo, a introdução de um protocolo de desmame reduziu significativamente a duração da ventilação mecânica. Embora, nosso resultado principal tenha sido diferente daquele apresentado por Randolph, os estudos são bem diferentes, no que diz respeito aos objetivos, metodologia e desenho. Uma das principais diferenças é que no estudo publicado por Randolph somente as crianças que não passaram no teste de respiração espontânea foram randomizadas para um dos 3 grupos de estudo, portanto, o teste de respiração espontânea foi utilizado como uma forma de selecionar aqueles pacientes com desmame mais difícil. 
A redução do tempo de ventilação mecânica em torno de 1 dia, com a introdução do protocolo de desmame, pode não parecer muito significativa. Porém, a mediana do tempo de ventilação foi reduzida de 4,7 dias para 3,5 dias o que representa uma redução de aproximadamente $25 \%$ do tempo total de ventilação mecânica. É importante enfatizar que embora o critério para realização do TRE seja diferente do critério para indicar extubação no grupo submetido a um desmame convencional, uma análise subsequente mostrou que não havia nenhuma diferença entre os dois grupos quanto aos parâmetros ventilatórios no momento da extubação (PEEP, PI, VC e $\mathrm{FiO}_{2}$ ) (tabela 5). Por tudo isso, não podemos afirmar, pelo presente estudo, que o paciente pode ser extubado mais precocemente se utilizarmos parâmetros ventilatórios mais altos como critério de extubação. Os critérios para o TRE foram mais liberais, porém eles não decidiam se o paciente ia ser extubado ou não, quem decidia a extubação era o TRE e efetivamente as crianças foram extubadas com parâmetros ventilatórios semelhantes. Por outro lado, $80 \%$ dos 20 pacientes submetidos a um teste de respiração espontânea com PEEP de $8 \mathrm{cmH}_{2} \mathrm{O}$, foram extubados com sucesso após um único teste de respiração espontânea. Isso pode sugerir que parâmetros ventilatórios mais altos não podem ser considerados como fator de risco para falha de extubação ou para necessidade de um segundo TRE. Nossa impressão é que as crianças do grupo controle ficaram mais tempo em ventilação mecânica do que realmente necessário. Provavelmente, se não criamos um mecanismo para reavaliar todos os dias se ainda existe a necessidade da ventilação, demoramos um pouco para perceber que o paciente já tem condições clínicas para ser extubado. 
É importante ressaltar que todas as análises do estudo foram realizadas baseadas no princípio de intenção de tratamento, assim, os pacientes foram analisados no grupo para o qual foram randomizados independente do tratamento que receberam. Poderia ser especulado se a exclusão dos 22 pacientes que tiveram um tratamento diferente daquele para o qual foram randomizados poderia trazer alguma mudança nos resultados do estudo. Porém, numa análise posterior isso não foi observado.

Também é importante ressaltar que não foram previstas análises de subgrupos e, portanto, nós decidimos não investigar se o protocolo seria mais eficiente em um determinado subgrupo de crianças baseado na idade, diagnóstico ou doença de base. Apesar de meramente especulativa, a nossa opinião é que, como os resultados foram positivos na nossa amostra, na qual a idade mediana das crianças é menor do que 1 ano e estudos prévios em adultos também positivos, contradizem uma relação entre o tratamento e a idade.

$\mathrm{Na}$ análise multivariada (tabela 3 ) observamos que o desenvolvimento de insuficiência renal aguda, pneumonia associada à ventilação mecânica ou SDRA durante a internação foi associado ao aumento do tempo em ventilação mecânica. No entanto, a proporção de pacientes com essas complicações foram semelhantes nos dois grupos. A associação de insuficiência renal aguda e duração do tempo de ventilação mecânica contrasta com estudo prévio $^{38}$, onde um balanço hídrico positivo não foi associado a maior tempo de ventilação mecânica e mostra a importância de maiores estudos prospectivos nesse campo. 
No grupo intervenção houve uma maior ocorrência de choque o que poderia ser associado a um maior tempo de ventilação mecânica, uma vez que um dos critérios para a realização da avaliação diária era estabilidade hemodinâmica. No entanto, essa condição clínica não foi associada com uma maior duração da ventilação mecânica na análise multivariada.

No grupo controle, embora não significante, houve uma maior ocorrência de crianças com doenças hepáticas o que poderia estar associado a um prolongamento do tempo de ventilação, devido a um aumento do volume abdominal e piora da mecânica respiratória associada a esse tipo de doença. Porém, a presença de hepatopatia não foi associada a uma maior duração da ventilação mecânica na análise multivariada. Talvez, a presença dessa condição clínica, possa justificar uma maior necessidade de ventilação não invasiva observada no grupo controle o que não era um desfecho esperado.

As avaliações diárias e o TRE são facilmente incorporáveis na rotina da UTI. No nosso estudo, o TRE foi realizado utilizando a modalidade pressão de suporte o que é mais fácil de implementar e gasta menos tempo do que o necessário para montar o sistema de tubo em T. Por outro lado, o tubo T pode ser utilizado em locais em que os recursos são mais escassos e não existe a disponibilidade de ventiladores com a modalidade pressão de suporte. Independente do sistema que for utilizado para realização do teste de respiração espontânea o nosso protocolo é uma estratégia fácil e eficiente para reduzir tempo de ventilação mecânica em pediatria. Esse é o primeiro protocolo de desmame, pelo menos que tenhamos conhecimento, 
em que o TRE foi utilizado como uma estratégia de desmame e realizado diariamente até o momento da extubação. Isso contrasta com os demais estudos onde o TRE foi utilizado somente nos pacientes em que o médico julgou como apto para extubação ou pronto a ser submetido a um teste de respiração espontânea ou, ainda, antes da randomização como no estudo realizado pela Randolph e colaboradores. ${ }^{27}$

Os resultados apresentados no nosso estudo são animadores, porém, o nosso protocolo tem algumas limitações. Em primeiro lugar, não avaliamos a via aérea superior uma vez que o TRE é realizado com crianças ainda intubadas. Sabemos que a Obstrução de Via Aérea Superior (OVAS) é uma das principais causas de falha de extubação em crianças. No nosso estudo, 33 crianças falharam na extubação, sendo que 57,5\% tiveram OVAS. O fato do nosso protocolo ter diminuído o tempo de extubação mecânica, não reduziu a taxa de falha de extubação. Isso mostra a importância de novos estudos enfatizando a via aérea superior. Além disso, o estudo não pode ser completamente cego apesar das nossas tentativas para diminuir viés. Esse tipo de desenho de estudo pode ter influenciado nos nossos resultados. 
6 Conclusões 
Em pacientes em ventilação mecânica, por mais de 24 horas, a realização de uma avaliação diária e teste de respiração espontânea foi capaz de reduzir o tempo de ventilação mecânica sem aumentar as taxas de reintubação ou a necessidade de ventilação não invasiva.

Não foi observado um aumento das complicações associadas ao prolongamento da VM. A incidência de pneumonia associada à VM e de extubação acidental foi semelhante nos dois grupos.

Algumas situações foram associadas a um aumento do tempo de VM tais como: a presença de Insuficiência Renal Aguda (IRA); apresentação de SDRA como diagnóstico que indicou a VM ou desenvolvimento da SDRA durante o período de VM; e desenvolvimento de pneumonia associada à VM. 
7 Anexos 


\section{Anexo 1 - Termo de consentimento livre e esclarecido do Hospital Universitário}

\section{TERMO DE CONSENTIMENTO LIVRE E ESCLARECIDO (TCLE)}

\section{DADOS DE IDENTIFICAÇÃO DO SUJEITO DA PESQUISA}

1. NOME DO PACIENTE:

DOCUMENTO DE IDENTIDADE:
DATA DE NASCIMENTO_L SEXO: M
ENDEREÇO:_
BAIRRO:
CEP:

2. RESPONSÁVEL LEGAL:

NATUREZA (grau de parentesco, tutor, curador etc.)

DOCUMENTO DE IDENTIDADE: SEXO: $M$

$\mathrm{F}$

DATA DE NASCIMENTO ___ CIDADE:
ENDEREÇO:
BAIRRO:
CEP:

Prezado(a) Senhor(a) estamos realizando um estudo que se chama "Efeito do Teste de Respiração Espontânea no Desmame de Ventilação Mecânica em Pediatria" e gostaríamos que seu(ua) filho(a) participe desse estudo.

Como já Ihe foi dito, seu filho(a) tem uma doença grave e está precisando de ajuda do aparelho para respirar. Quando ele(a) estiver melhor este aparelho será lentamente retirado. Existem algumas formas de se proceder a essa retirada.

Com o objetivo de tentar diminuir o tempo em que seu filho(a) irá permanecer no aparelho para respirar, iremos comparar duas formas de retirada do aparelho.Uma delas é a que habitualmente é realizada na unidade e na segunda, antes da retirada do tubo, faremos um teste para observar a capacidade dele(a) respirar sozinho(a) com mínima ajuda do aparelho.

As crianças serão sorteadas para participar de um dos dois grupos. Caso seu filho(a) seja sorteado(a) para o grupo do teste ele(a) continuará ligado(a) ao aparelho, porém com mínima ajuda por um período de cerca de 2 horas enquanto será observado atentamente qualquer sinal de cansaço.

Esse teste não implica em nenhum risco adicional. Se ele(a) ficar cansado(a) durante o teste, este será interrompido imediatamente e ele(a) retornará a situação prévia. Se ele(a) ficar bem durante o teste ele(a) será imediatamente retirado(a) do aparelho. Com esse teste esperamos diminuir o tempo que seu filho(a) permanece conectado(a) ao aparelho.

Se esse teste for eficiente, a retirada do respirador pode se processar de maneira mais rápida no futuro podendo ajudar diretamente inúmeras crianças. 
Todos os dados e resultados coletados durante a pesquisa serão sigilosos, porém com total acesso aos responsáveis. Não haverá nenhum custo financeiro relacionado á participação na pesquisa, e também não haverá qualquer tipo de pagamento para a participação do(a) seu(ua) filho(a) no estudo.

Você poderá esclarecer suas dúvidas com os membros da equipe e não aceitar participar da pesquisa. Poderá também retirar seu(ua) filho(a) da pesquisa a qualquer momento e, portanto, deixar de participar do estudo sem que em nenhuma opção o seu atendimento no hospital ficará prejudicado.

Sua assinatura nesse termo de consentimento indica que você decidiu autorizar a participação do(a) seu(ua) filho(a) no estudo, após leitura (ou alguém ter lido para você) das informações colocadas nesse documento.

Declaro que, após convenientemente esclarecido pelo pesquisador e ter entendido o que me foi explicado, consinto em participar do presente Projeto de Pesquisa.

São Paulo, de de 20

\section{INFORMAÇÕES DE NOMES, ENDEREÇOS E TELEFONES DOS RESPONSÁVEIS PELO ACOMPANHAMENTO DA PESQUISA, PARA CONTATO EM CASO DE INTERCORRÊNCIAS CLÍNICAS E REAÇÕES ADVERSAS.}

Flávia Andréa Krepel Foronda - R. Do Chá ,n² 21 apto. 51 - Tel: 37550945/ 99117315/31550888

Identificação do CEP-HU: Endereço: Av. Prof. Lineu Prestes, 2565 - Cidade Universitária - CEP: 05508-900 - São Paulo - SP - Telefones: 3039-9457 ou 3039-9479 - E-mail: cep@hu.usp.br. 


\section{Anexo 2 - Termo de consentimento livre e esclarecido do Instituto da Criança do Hospital das Clínicas}

\section{HOSPITAL DAS CLÍNICAS}

DA

FACULDADE DE MEDICINA DA UNIVERSIDADE DE SÃO PAULO

\section{TERMO DE CONSENTIMENTO LIVRE E ESCLARECIDO}

\begin{tabular}{l}
\hline I - DADOS DE IDENTIFICAÇÃO DO SUJEITO DA PESQUISA \\
1. NOME DO PACIENTE: \\
DOCUMENTO DE IDENTIDADE: \\
DATA DE NASCIMENTO_ SEXO: M \\
ENDEREÇO: \\
BAIRRO:_ CIELEFONE: _ CIDADE: _ _ APTO: \\
CEP:
\end{tabular}

2. RESPONSÁVEL LEGAL:

NATUREZA (grau de parentesco, tutor, curador etc.)

DOCUMENTO DE IDENTIDADE: SEXO: $M$

$\mathrm{F}$

DATA DE NASCIMENTO

1

ENDEREÇO:

$\mathrm{N}^{\mathrm{o}}$ : APTO:

BAIRRO

CEP: CIDADE: TELEFONE:

\section{II - DADOS SOBRE A PESQUISA CIENTÍFICA}

1. TÍTULO DO PROTOCOLO DE PESQUISA: Efeito do Teste de Respiração Espontânea no Desmame de Ventilação Mecânica em Pediatria.

2. PESQUISADOR: Eduardo Juan Troster

CARGO/FUNÇÃO: Médico

UNIDADE DO HCFMUSP: Centro de Terapia Intensiva Pediátrica

3. AVALIAÇÃO DO RISCO DA PESQUISA:

MÉdIO SEM RISCO RISCO MÍNIMO X RISCO
RISCO BAIXO
RISCO MAIOR

(probabilidade de que o indivíduo sofra algum dano como consequência imediata ou tardia do estudo)

4.DURAÇÃO DA PESQUISA : Cerca de dois anos. 


\section{III - REGISTRO DAS EXPLICAÇÕES DO PESQUISADOR AO PACIENTE OU SEU REPRESENTANTE LEGAL SOBRE A PESQUISA, CONSIGNANDO:}

Prezado(a) Senhor(a) como já lhe foi dito, seu filho(a) tem uma doença grave e está precisando de ajuda do aparelho para respirar. Quando ele(a) estiver melhor este aparelho será lentamente retirado. Existem algumas formas de se proceder a essa retirada.

Com o objetivo de tentar diminuir o tempo em que seu filho(a) irá permanecer no aparelho para respirar, iremos comparar duas formas de retirada do aparelho.Uma delas é a que habitualmente é realizada na unidade e na segunda, antes da retirada da cânula, faremos um teste para observar a capacidade dele(a) respirar sozinho(a) com mínima ajuda do aparelho.

As crianças serão sorteadas para participar de um dos dois grupos. Caso seu filho(a) seja sorteado(a) para o grupo do teste ele(a) continuará ligado ao aparelho, porém com mínima ajuda por um período de cerca de 2 horas enquanto será observado atentamente qualquer sinal de cansaço.

Esse teste não implica em nenhum risco adicional. Se ele(a) ficar cansado(a) durante o teste, este será interrompido imediatamente e ele(a) retornará a situação prévia. Se ele(a) ficar bem durante o teste ele(a) será imediatamente retirado(a) do aparelho. Com esse teste esperamos diminuir o tempo que seu filho(a) permanece conectado(a) ao aparelho.

Se esse teste for eficiente, a retirada do respirador pode se processar de maneira mais rápida no futuro podendo ajudar diretamente inúmeras crianças.

\section{IV - ESCLARECIMENTOS DADOS PELO PESQUISADOR SOBRE GARANTIAS DO SUJEITO DA PESQUISA:}

Todos os dados e resultados coletados durante a pesquisa serão sigilosos, porém com total acesso aos responsáveis. Não haverá nenhum custo financeiro relacionado á participação na pesquisa, e também não haverá qualquer tipo de pagamento para a participação do(a) seu(ua) filho(a) no estudo.

Você poderá esclarecer suas dúvidas com os membros da equipe e poderá não aceitar participar da pesquisa sem que isso cause prejuízo no seu atendimento no hospital.

Sua assinatura nesse termo de consentimento indica que você decidiu autorizar a participação do(a) seu(ua) filho(a) no estudo, após leitura (ou alguém ter lido para você) das informações colocadas nesse documento.

\section{V - INFORMAÇÕES DE NOMES, ENDERECCOS E TELEFONES DOS RESPONSÁVEIS PELO ACOMPANHAMENTO DA PESQUISA, PARA CONTATO EM CASO DE INTERCORRÊNCIAS CLÍNICAS E REAÇÕES ADVERSAS.}

Flávia Andréa Krepel Foronda - R. Do Chá ,nº 21 apto. 51 - Tel: 37550945/ 99117315/ 31550732

\section{VI - OBSERVAÇÕES COMPLEMENTARES:}

\section{VII - CONSENTIMENTO PÓS-ESCLARECIDO}

Declaro que, após convenientemente esclarecido pelo pesquisador e ter entendido o que me foi explicado, consinto em participar do presente Protocolo de Pesquisa

São Paulo, de de 2 


\section{Anexo 3 - Avaliação Diária}

\section{Avaliação Diária}

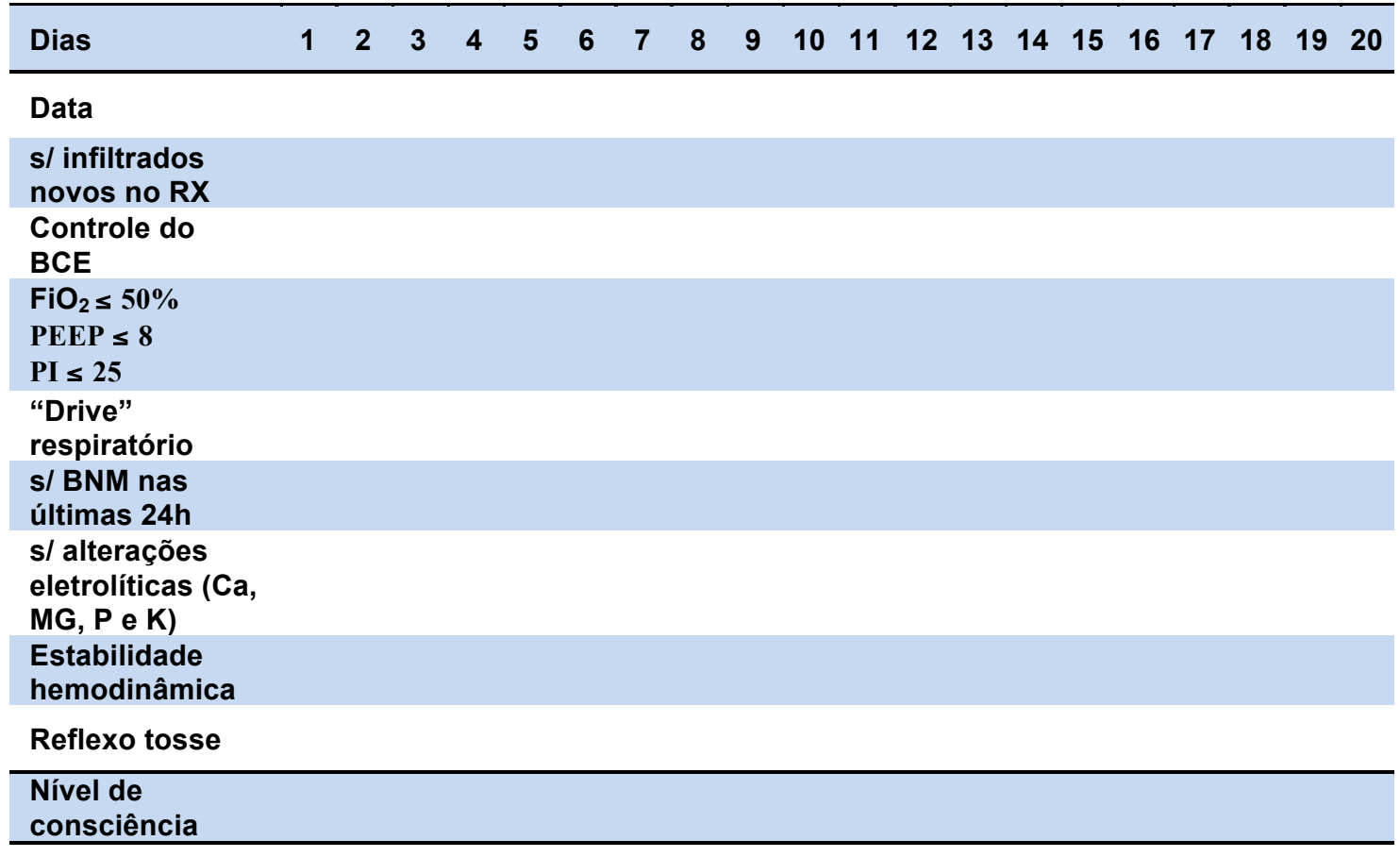

$\mathrm{Hb} \geq 8 \mathrm{mg} / \mathrm{dL}$

FINAL 


\section{Anexo 4 - Escala de COMFORT-Behavior ${ }^{39}$}

Escala: Comfort-Behavior

\begin{tabular}{|c|c|c|c|c|c|}
\hline NOTA & 1 & 2 & 3 & 4 & 5 \\
\hline $\begin{array}{l}\text { Movimento } \\
\text { Físico }\end{array}$ & s/ Movimentos & $\begin{array}{l}\text { Movimentos } \\
\text { leves } \\
\text { ocasionais }\end{array}$ & $\begin{array}{l}\text { Movimentos } \\
\text { leves } \\
\text { frequêntes }\end{array}$ & $\begin{array}{l}\text { Movimentos } \\
\text { vigorosos em } \\
\text { extremidades }\end{array}$ & $\begin{array}{l}\text { Movimentos } \\
\text { vigorosos em } \\
\text { dorso e } \\
\text { cabeça }\end{array}$ \\
\hline Tônus Muscular & $\begin{array}{l}\text { Totalmente } \\
\text { relaxado }\end{array}$ & $\begin{array}{l}\downarrow \text { Tônus } \\
\text { muscular }\end{array}$ & $\begin{array}{l}\text { Tônus } \\
\text { muscular } \\
\text { normal }\end{array}$ & $\begin{array}{l}\uparrow \text { Tônus cl } \\
\text { flexão de } \\
\text { dedos }\end{array}$ & $\begin{array}{l}\text { Rigidez } \\
\text { muscular }\end{array}$ \\
\hline Prontidão & $\begin{array}{l}\text { Profundamente } \\
\text { adormecido }\end{array}$ & $\begin{array}{l}\text { Levemente } \\
\text { adormecido }\end{array}$ & Sonolento & $\begin{array}{l}\text { Acordado e } \\
\text { alerta }\end{array}$ & Hiperreativo \\
\hline $\begin{array}{l}\text { Tranquilidade } \\
\text { ou Agitação }\end{array}$ & Calmo & $\begin{array}{l}\text { Pouco } \\
\text { ansioso }\end{array}$ & Ansioso & Muito ansioso & Pânico \\
\hline Respiração & $\begin{array}{l}\text { s/ Tosse e s/ } \\
\text { respiração } \\
\text { espontânea }\end{array}$ & $\begin{array}{l}\text { Respiração } \\
\text { espontânea s/ } \\
\text { resistência ao } \\
\text { ventilador }\end{array}$ & $\begin{array}{c}\text { Tosse } \\
\text { ocasional ou } \\
\text { resistência } \\
\text { ao } \\
\text { ventilador }\end{array}$ & $\begin{array}{c}\text { Tosse regular } \\
\text { ou respiração } \\
\text { ativa contra o } \\
\text { ventilador }\end{array}$ & $\begin{array}{l}\text { Briga } \mathrm{c} / \mathrm{o} \\
\text { ventilador }\end{array}$ \\
\hline Tensão Facial & $\begin{array}{l}\text { Músculos } \\
\text { faciais } \\
\text { relaxados }\end{array}$ & $\begin{array}{c}\text { Tônus facial } \\
\text { normal }\end{array}$ & $\begin{array}{l}\text { Tensão } \\
\text { evidente em } \\
\text { alguns } \\
\text { músculos }\end{array}$ & $\begin{array}{l}\text { Tensão } \\
\text { evidente em } \\
\text { todos os } \\
\text { músculos }\end{array}$ & $\begin{array}{l}\text { Músculos } \\
\text { faciais } \\
\text { contorcidos } \\
\text { "careta" }\end{array}$ \\
\hline FINAL & & & & & \\
\hline
\end{tabular}

Valor: Ideal $=11-22 \quad$ Aceitável $\geq 9$ 


\section{Anexo 5 - Frequência Respiratória normal em crianças ${ }^{40}$}

\begin{tabular}{lc}
\hline Idade (anos) & $\begin{array}{c}\text { Frequência } \\
\text { (respiração por minuto) }\end{array}$ \\
\hline$<1$ & $30-60$ \\
\hline a 3 & $24-40$ \\
4 a 5 & $22-34$ \\
6 a 12 & $18-30$ \\
13 a 18 & $12-18$ \\
\hline
\end{tabular}

Frequência respiratória acima de 60 é anormal e é sinal de alerta em qualquer idade 


\section{Anexo 6 - Publicação}

\section{The impact of daily evaluation and spontaneous breathing test on the duration of pediatric mechanical ventilation: A randomized controlled trial*}

Flávia K. Foronda, MD; Eduardo J. Troster, MD, PhD; Julio A. Farias, MD; Carmen S. Barbas, MD, PhD; Alexandre A. Ferraro, MD, PhD; Lucília S. Faria, MD; Albert Bousso, MD, PhD; Flávia F. Panico, MD; Artur F. Delgado, MD, PhD

Objectives: To assess whether the combination of daily evaluation and use of a spontaneous breathing test could shorten the duration of mechanical ventilation as compared with weaning based on our standard of care. Secondary outcome measures included extubation failure rate and the need for noninvasive ventilation.

Design: A prospective, randomized controlled trial.

Setting: Two pediatric intensive care units at university hospitals in Brazil.

Patients: The trial involved children between 28 days and 15 yrs of age who were receiving mechanical ventilation for at least $24 \mathrm{hrs}$. Interventions: Patients were randomly assigned to one of two weaning protocols. In the test group, the children underwent a daily evaluation to check readiness for weaning with a spontaneous breathing test with $10 \mathrm{~cm} \mathrm{H}_{2} \mathrm{O}$ pressure support and a positive end-expiratory pressure of $5 \mathrm{~cm} \mathrm{H}_{2} \mathrm{O}$ for 2 hrs. The spontaneous breathing test was repeated the next day for children who failed it. In the control group, weaning was performed according to standard care procedures.
Measurements and Main Results: A total of 294 eligible children were randomized, with 155 to the test group and 139 to the control group. The time to extubation was shorter in the test group, where the median mechanical ventilation duration was $\mathbf{3 . 5}$ days ( $95 \%$ confidence interval, 3.0 to 4.0 ) as compared to 4.7 days (95\% confidence interval, 4.1 to 5.3 ) in the control group ( $p=$ .0127). This significant reduction in the mechanical ventilation duration for the intervention group was not associated with increased rates of extubation failure or noninvasive ventilation. It represents a $30 \%$ reduction in the risk of remaining on mechanical ventilation (hazard ratio: 0.70 ).

Conclusions: A daily evaluation to check readiness for weaning combined with a spontaneous breathing test reduced the mechanical ventilation duration for children on mechanical ventilation for $>\mathbf{2 4}$ hrs, without increasing the extubation failure rate or the need for noninvasive ventilation. (Crit Care Med 2011; 39:2526-2533)

KEY WoRDS: artificial, respiration; intensive care units; pediatrics; randomized controlled trial; respiratory insufficiency; ventilator weaning
A cute respiratory insufficiency is common in the pediatric population. It is estimated that two to three million children a yea worldwide die of respiratory causes (1)

Undoubtedly, mechanical ventilation

(MV) is an important medical intervention

*See also p. 2581.

From the Intensive Care Unit (FKF, LSF), Hospital Sírio Libanês, São Paulo, Brazil; Pediatric Department (FKF EJT, AAF, LSF, FFP, AFD), and Pneumology Department (CSB), Hospital das Clínicas, Medical School of São Paulo University, São Paulo, Brazil; Intensive Care Unit (EJT, AB, FFP), Hospital Albert Einstein, São Paulo, Brazil; Intensive Care Unit (JAF), Hospital de Niños R Gutiérrez, Buenos Aires University, Buenos Aires, Argentina; and Intensive Care Unit (AB), Hospital Universitário, Medical School of São Paulo University, São Paulo, Brazil.

The authors have not disclosed any potential conticts of interest.

For information regarding this article, E-mail: flikrepel@foronda.com.br

Copyright (๑) 2011 by the Society of Critical Care Medicine and Lippincott Williams \& Wilkins

DOI: 10.1097/CCM.0b013e3182257520 capable of reducing morbidity and mortality in this population, especially among severe cases (2). However, it remains a practical challenge to accurately predict when a patient is ready to sustain spontaneous breathing and successfully discontinue MV (2-5).

Extubation failure, which is defined as the need for reintubation and reestablishment of MV within 48 to 72 hrs after the racheal cannula is removed $(6-10)$, is associated with a poor prognosis and an increased mortality rate $(11,12)$. In the pediatric population, extubation failure rates vary between $16 \%$ and $19 \%(6-9)$. Nevertheless, ventilation times are often extended unnecessarily. In a previous study in which 136 children underwent unplanned extubation, most of the patients did not require reintubation (12).

Extended MV periods are associated with an increased risk of complications, such as nosocomial pneumonia $(13-16)$, ventilator-induced lung injury $(17,18)$, airway injuries (19), and an increased need for sedatives. Thus, MV should be discontinued as soon as possible.

However, successful early extubation is difficult because extubation criteria may vary. Thus, the continued search for criteria to indicate the correct time to end MV is a priority. Several previous studies conducted in the pediatric population have tried to define predictors of successful extubation. However, it has not been possible to determine which set of parameters accurately predicts successful extubation $(6,8,9,20)$.

The spontaneous breathing test (SBT) was developed as an attempt to identify patients who are ready to discontinue ventilation $(21,22)$. The test aims at monitoring signs of respiratory muscle fatigue while the patient is still intubated. A previous study showed that SBT performed with a T-piece was able to predict successful extubation in $70 \%$ of intubated children (20). In a subsequent work, the 
same research team showed that SBT performed with a T-piece or pressure support was equally effective in identifying these patients, with both predicting the same extubation failure rates (11).

In adults, the use of daily evaluation combined with an SBT has been shown to reduce MV duration, treatment costs, and treatment-associated complications (23).

Some authors $(2,24,25)$ have proposed a combination of daily evaluation and SBT use as a possible way to reduce ventilation times. However, to our knowledge, this strategy has not been formally investigated in the pediatric population. We conducted a randomized controlled trial to determine whether the combination of a daily evaluation and SBT use could shorten the MV duration as compared with weaning based on standard care procedures in a pediatric population. We also determined the extubation failure rate, the need for postextubation noninvasive ventilation (NIV), the occurrence of ventilator-associated pneumonia and accidental extubation, and the factors associated with MV duration.

\section{METHODS}

\section{Patients}

This study was approved by the institutional review boards of the participating hospitals, and written informed consent was obtained from the parents or guardians of all patients before enrollment.

Eligible patients were children of both genders, aged between 28 days and 15 yrs, who were admitted to the pediatric intensive care units (ICUs) of the Hospital das Clínicas da Universidade de São Paulo and Hospital Universitário in Sao Paulo, Brazil, between July 2007 and July 2009 , and who had been receiving MV for $>24$ hrs. The exclusion criteria were as follows: intubation due to upper airway obstruction (UAO), the presence of diaphragmatic hernia or paralysis; chronic MV use; cyanotic congenital heart disease; primary pulmonary hypertension; neuromuscular disease; or tracheostomy. Patients on chronic MV were defined as those who were dependent on invasive or noninvasive ventilation before ICU admission, including children from other units at the same hospital, other hospitals, or home-care units. Patients could be enrolled in the study only once during their hospital stay, and only the first intubation was considered for analysis in patients who required reintubation. The study is registered in the International Standard Randomized Controlled Trial Number Register (ISRCTN37806223).

\section{Overall Study Design}

The design of this study is illustrated in Figure 1. Patients were randomly assigned to

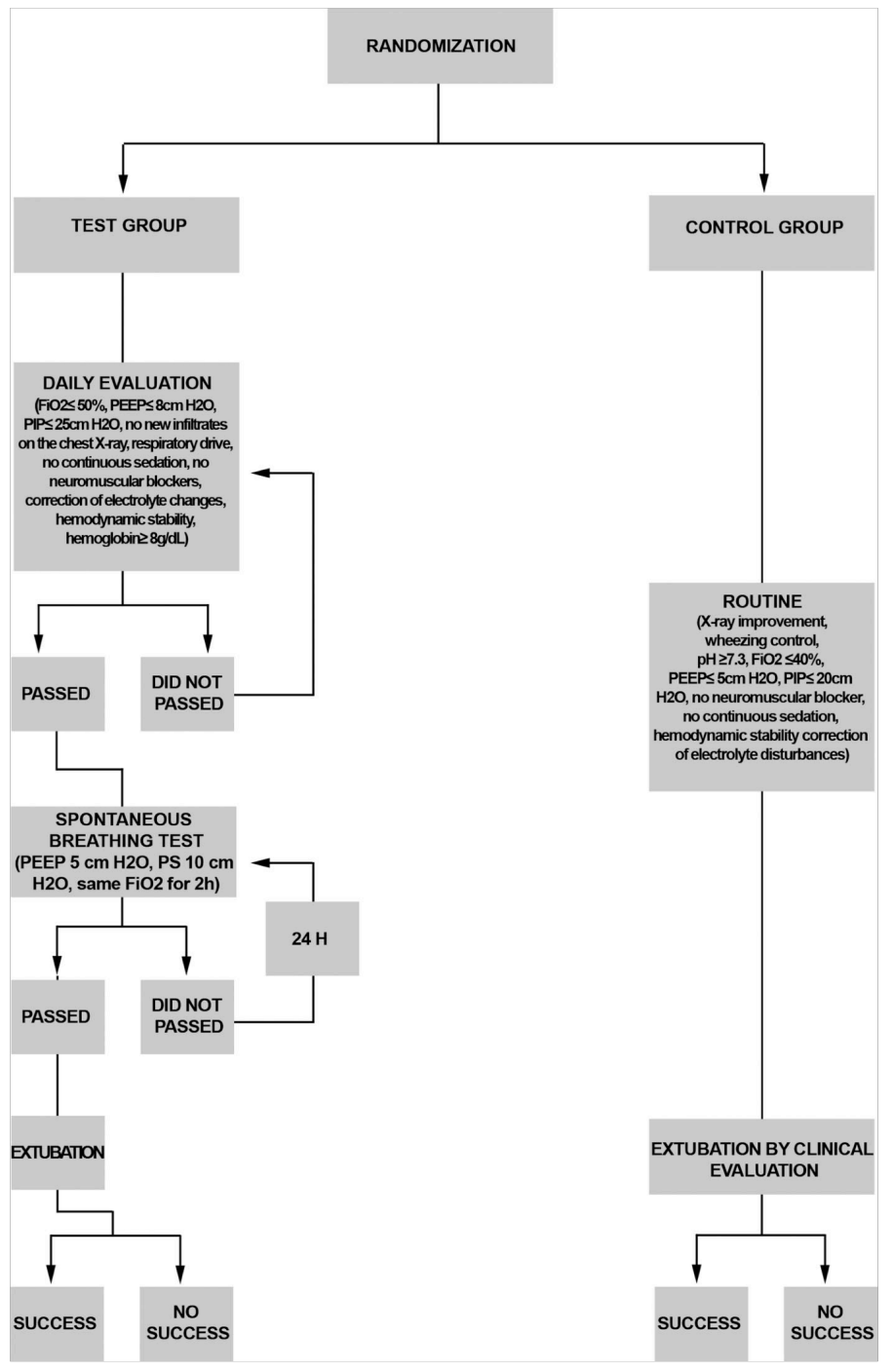

Figure 1. Study design. PEEP, positive end-expiratory pressure; PIP, peak inspiratory pressure; $P S$, pressure support

weaning following a protocol that combined the daily screening of SBT (test group), or weaning based on standard care procedures (contro group). In the test group, the SBT was performed on patients after they fulfilled predetermined criteria on the daily evaluation. This procedure was performed every morning until the patient was extubated by fellows who were specifically trained for this task before study initiation, and then every 3 months; these fellows were not involved in the decision to extubate patients. In addition, new cases from both participating institutions were evaluated once a week to check whether the eligibility criteria had been followed at enrollment and whether the daily evaluations were being performed appropriately. The two study groups were treated by the same medical team, and team members were unaware of the group to which the patients had been randomized until the patients passed the daily evaluation and the SBT was indicated. In the control group, weaning was performed at the 
discretion of the attending physician in charge of the medical team, with no influence from study protocols. The ventilators used in the study were the Newport E500 (Newport Medical Instruments, Newport Beach, CA), Servoi (Siemens, Elema, Solna, Sweden), and Evita 4 (Dräger, Lübeck, Germany).

To control for the differences between routine procedures at each ICU, randomization was stratified according to hospital and carried out using opaque plastic bags containing varying numbers (i.e., six, 12 , or 20 ) of sealed envelopes prepared by staff who were not involved with patient care. The same fellow in charge of the daily evaluations, with no knowledge from the medical team, randomized patients in a 1:1 ratio.

\section{Study Procedures}

To pass the daily evaluation and undergo the SBT, patients had to meet the following criteria: $\mathrm{FIO}_{2} \leq 50 \%$; positive end-expiratory pressure $(\mathrm{PEEP}) \leq 8 \mathrm{~cm} \mathrm{H}_{2} \mathrm{O}$; peak inspiratory pressure (PIP) $\leq 25 \mathrm{~cm} \mathrm{H}_{2} \mathrm{O}$; an absence of new infiltrates on the chest radiograph; the presence of respiratory drive; no continuous sedation; no use of neuromuscular blockers in the last $24 \mathrm{hrs}$; correction of electrolyte changes (calcium, magnesium, phosphorus, and potassium); hemodynamic stability (doses of sodium nitroprusside, dopamine, or dobutamine $<10 \mu \mathrm{g} / \mathrm{kg} / \mathrm{min}$ ); and hemoglobin $\geq 8$ $\mathrm{g} / \mathrm{dL}$. Patients who met these criteria underwent SBT with a PEEP of $5 \mathrm{~cm} \mathrm{H}_{2} \mathrm{O}$, pressure support ventilation of $10 \mathrm{~cm} \mathrm{H}_{2} \mathrm{O}$, and the $\mathrm{FIO}_{2}$ that was used before the test for a period of 2 hrs. The test was interrupted in the presence of any signs of intolerance, including respiratory or heart rate increase $20 \%$ above the initial values, signs of increased respiratory work (i.e., use of accessory muscles or paradoxic respiration), changes in the level of consciousness, blood pressure $<$ fifth percentile for patient's age, arterial blood oxygen saturation $<90 \%$, or $\mathrm{PaCO}_{2}>50 \mathrm{~mm} \mathrm{Hg}$ (or an increase $>10 \mathrm{~mm} \mathrm{Hg}$ in $1 \mathrm{hr}$ in patients with chronic lung disease). Arterial blood gas samples were collected before the SBT and in the first hour of the test. Extubation was performed in patients who did not meet any criteria for a failed test. Otherwise, MV was reestablished using the previous parameters, and the test was repeated $24 \mathrm{hrs}$ later (Fig. 1).

In the control group, weaning was performed according to the routine procedures of each ICU, with no SBT. Briefly, the ventilato mode and settings were at the discretion of the attending physician. Pressure-based ventilatory modes were more frequently used in our institution, although other modes were available. The most frequently used modes were pressure support, synchronized intermittent mandatory ventilation, and pressure-controlled ventilation, the latter of which was used more often in severe cases (26). Respiratory frequency and PIP reductions were performed according to the presence of ventilatory parameters, including increased chest cage expansion, increased exhaled tidal volume, and reduced $\mathrm{PaCO}_{2}$ or end-tidal $\mathrm{CO}_{2}$. FIO and PEEP were reduced according to patient oxygenation to maintain arterial oxygen saturation between $92 \%$ and $98 \%$.

\section{Weaning and Extubation}

Until patients in the test group met the criteria for the SBT, weaning was similar to that of the control group and was initiated according to clinical and blood-gas criteria. The criteria routinely used in the two ICUs for extubating patients from MV included the following: resolution of the cause that led to intubation, pharmacologic control of wheezing, absence of respiratory acidosis $(\mathrm{pH}<7.3), \mathrm{FIO}_{2} \leq 40 \%$, PEEP $\leq 5 \mathrm{~cm} \mathrm{H}_{2} \mathrm{O}$, PIP $<20 \mathrm{~cm} \mathrm{H}_{2} \mathrm{O}$, body temperature $<38.5^{\circ} \mathrm{C}$, no need for continuous sedation, hemodynamic stability, correction of electrolyte disturbances, and the absence of a neuromuscular blocker in the last $24 \mathrm{hrs}$.

After extubation, patient progress was monitored for $48 \mathrm{hrs}$. A failed extubation was defined as the need for reintubation within this period. Reintubation was indicated when patients met two or more clinical criteria (i.e., increase in respiratory rate $\geq 40 \%$ of the normal rate for age, apnea $>20$ secs, subdiaphragmatic or suprasternal retractions, or cyanosis) or blood-gas criteria $\left(\mathrm{PaO}_{2} / \mathrm{FIO}_{2}\right.$ ratio $<200$ or $\mathrm{PacO}_{2}>50 \mathrm{~mm}$ $\mathrm{Hg}$ with $\mathrm{pH}<7.35$ ). NIV was indicated in cases of respiratory distress, hypoxemia (pulse oxygen saturation $<90 \%$ ), and decreased level of consciousness leading to inadequate respiratory ef fort, UAO, or cardiovascular insufficiency. Patients were reintubated and placed on $\mathrm{MV}$ if they were unable to maintain $\mathrm{PaO}_{2} \geq 80 \mathrm{~mm} \mathrm{Hg}$ with $\mathrm{FiO}_{2} \leq 60 \%$ after an attempt at NIV with two different levels of pressure.

\section{Data Collection and Statistical \\ Analysis}

For all patients, the following variables were collected and analyzed: age, gender, primary diagnosis, comorbidities, indication for MV, Pediatric Risk of Mortality II score, Pediatric Index of Mortality 2, respiratory complications, duration of MV, ventilatory parameters, medication use, extubation failure, and use of NIV.

For sample size calculation, given our previous experience at the participating ICUs, the inclusion of 133 patients per group would give the study a power of $80 \%$ in detecting an increase in the proportion of patients successfully extubated at 4 days, from $35 \%$ in the control group to $50 \%$ in the test group based on a one-sided Type I error of 5\%. The study was analyzed on an intention-to-treat basis.
The Kolmogorov-Smirnov test was used to assess whether continuous variables were normally distributed. Continuous variables with non-normal distribution were summarized as medians and interquartile ranges, and comparisons between groups were conducted using the Mann-Whitney $U$ test. For categorical data, percentages and their $95 \%$ confidence intervals were calculated, and comparisons between groups were conducted using Pearson's chisquared test or Fisher's exact test, as appropriate.

Kaplan-Meier survival curves were used to estimate the time to extubation in both groups, with comparisons based on the logrank test. Time to extubation was defined as the elapsed time between the initiation of ventilation and the first extubation. Only patient who were extubated were considered for the analysis of MV duration and for the analysis of the secondary outcomes. Those who died without ever being weaned from the ventilator were not considered for the analysis of these outcomes. A 30-day follow-up period was considered for analysis. Patients on MV for $>30$ days were considered as weaning failures and thus censored in the analysis. The Cox proportional-hazards regression model was used to investigate the predictive role of variables with $p<.20$ using univariate analyses.

Statistical analysis was conducted by a professional research organization and performed using SPSS software, version 17. Two-sided $p$ values $<.05$ were considered significant.

\section{RESULTS}

\section{Patient Characteristics}

A total of 776 patients were screened, but 464 were considered ineligible for the study before randomization (Fig. 2). O the 312 children who were randomized to the study, 18 were not eligible to participate; 11 patients were on ventilation for $<24$ hrs, three had neuromuscular disease, two had UAO, a single patient was older than $15 \mathrm{yrs}$, and for another patient, the index intubation was the second intubation. Eight of these protocol violations occurred in the test group and ten in the control group (Fig. 2). These ineligible patients were therefore not included in the analysis.

Of the 294 eligible children randomized to the study, 155 were assigned to the test group and 139 were assigned to the control group. Patients had a median age of 11 months, and $54.8 \%$ were male. Table summarizes demographic and clinical baseline characteristics of these patients The groups did not differ in terms of age, gender, Pediatric Risk of Mortality II and Pediatric Index of Mortality 2 scores, or primary diagnoses, with the exception of 


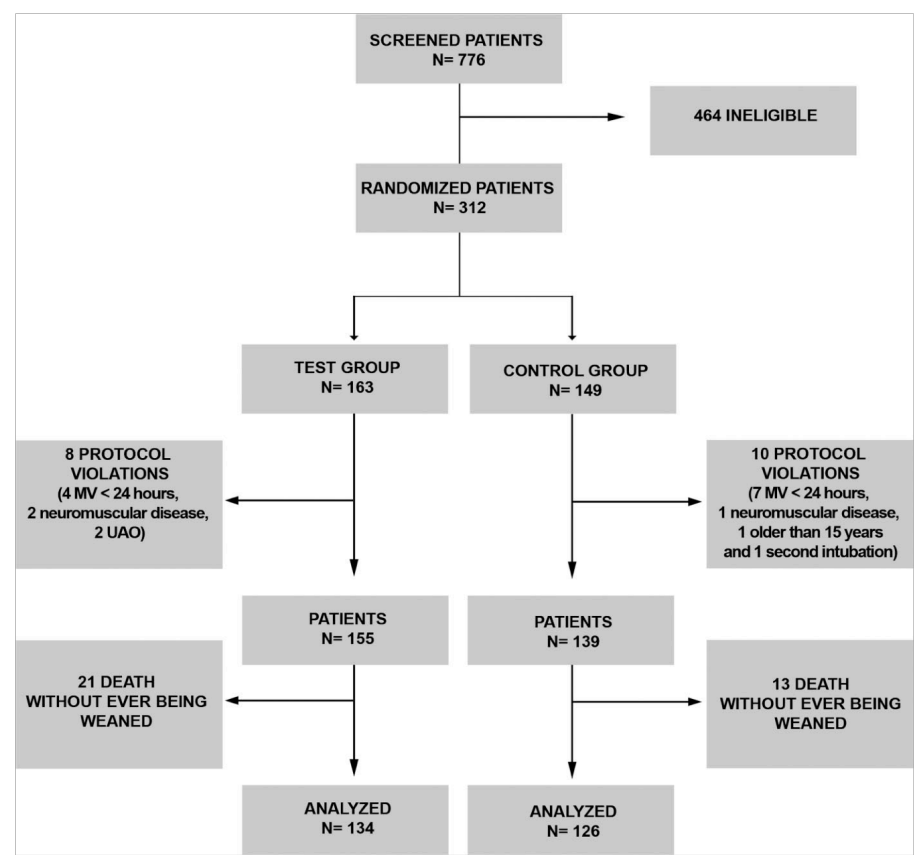

Figure 2. Patient flow. $M V$, mechanical ventilation; $U A O$, upper airway obstruction.

Table 1. Demographic and clinical baseline characteristics of the patients enrolled according to the study group

\begin{tabular}{lccc}
\hline \multicolumn{1}{c}{ Characteristics } & $\begin{array}{c}\text { Test Group } \\
(\mathrm{n}=155)\end{array}$ & $\begin{array}{c}\text { Control Group } \\
(\mathrm{n}=139)\end{array}$ & $p$ \\
\hline Age $\left(\mathrm{yrs}^{a}\right.$ & $0.922[0.303 ; 3.483]$ & $0.933[0.344 ; 3.399]$ & .918 \\
Pediatric Risk of Mortality II $^{a}$ & $0.050[0.025 ; 0.118]$ & $0.051[0.128 ; 0.275]$ & .756 \\
${\text { Pediatric Index of Mortality } 2^{a}}_{\text {Male }^{b}}$ & $0.098[0.040 ; 0.244]$ & $0.080[0.036 ; 0.177]$ & .148 \\
Immunodeficiency $^{b}$ & $86(55.5 \%)$ & $75(54 \%)$ & .793 \\
Congenital Heart Disease $^{b}$ & $7(4.5 \%)$ & $6(4.3 \%)$ & .934 \\
Genetic Disorders $^{b}$ & $9(5.8 \%)$ & $8(5.8 \%)$ & .985 \\
Malignant Tumors $^{b}$ & $7(4.5 \%)$ & $7(5 \%)$ & .834 \\
Neurologic Disorders $^{b}$ & $12(7.7 \%)$ & $3(2.2 \%)$ & .030 \\
Liver Disease $^{b}$ & $17(11 \%)$ & $9(6.5 \%)$ & .176 \\
Chronic Lung Disease $^{b}$ & $13(8.4 \%)$ & $20(14.4 \%)$ & .104 \\
Leukemia $^{b}$ & $20(12.9 \%)$ & $19(13.7 \%)$ & .847 \\
Main Indications for Mechanical Ventilation $^{b}$ & $5(3.2 \%)$ & $6(4.3 \%)$ & .623 \\
Congestive heart failure $^{b}$ & $4(2.6 \%)$ & $9(6.5 \%)$ & .105 \\
Wheezing $^{b}$ & $34(21.9 \%)$ & $31(22.3 \%)$ & .940 \\
Septic shock $^{b}$ & $35(22.6 \%)$ & $20(14.4 \%)$ & .072 \\
Coma $^{b}$ & $24(15.5 \%)$ & $14(10.1 \%)$ & .167 \\
Pneumonia $^{b}$ & $60(38.7 \%)$ & $60(43.2 \%)$ & .576 \\
Bronchiolitis $^{b}$ & $27(17.4 \%)$ & $34(24.5 \%)$ & .200 \\
Acute respiratory distress syndrome $^{b}$ & $13(8.4 \%)$ & $8(5.7 \%)$ & .382 \\
\hline
\end{tabular}

Values are median [25th percentile and 75 th percentile] or numbers (percent).

${ }^{a}$ Mann-Whitney $U$ test; ${ }^{b}$ Chi-square test.

malignant tumors, which showed a higher frequency in the intervention group. In addition, we found no significant differences between the test and control groups re-

garding the main indications for MV. Finally, there were no significant differences between the two groups regarding the mortality rate observed during the study course
(14.8\% in the test group vs. $10.8 \%$ in the control group; $p=.302$ ).

As the primary and secondary objectives of the study were focused on patients that had been extubated or were still on MV during the 30-day follow-up period, 34 patients who died without ever being weaned from the ventilator were not considered for the following analysis; 21 were in the test group and 13 were in the control group (Fig. 2).

In the test group, five patients were extubated without undergoing a complete SBT, including three due to the physician's decision, one due to psychomotor agitation during the test, and one due to accidental extubation. In the control group, 17 patients were extubated after a SBT; most cases occurred within the last 2 months of the study, but all patients were analyzed on an intention-to-treat basis. Table 2 shows the demographic and clinical baseline characteristics of the patients who were considered for the analysis of the primary and secondary outcomes.

In both groups, ICU admission was mostly due to medical reasons $(92.9 \%$ in the control group vs. $96.3 \%$ in the test group; $p=.223$ ). The main indications for initiating MV were pneumonia (42.3\%), wheezing $(24.6 \%)$, bronchiolitis $(23.0 \%)$, septic shock (16.9\%), and coma (12.3\%). Acute respiratory distress syndrome (ARDS) was present in 5\% of the patients. We found no significant differences between the test and control groups regarding these main indications for MV (Table 2)

Comparing the occurrence of complications in both groups, the frequency of shock during MV was higher in the test group than in the control group $(12.7 \%$ vs. $5.6 \% ; p=.047)$. For the rates of all other complications analyzed, namely ARDS ( $p=$ $.585)$, sepsis $(p=.218)$, acute renal failure (ARF) $(p=.190)$, and coagulopathy ( $p=$ .806), no significant between-group differences were found. The two groups were also similar with respect to MV modes. Pressure-controlled modes (i.e., pressurecontrolled ventilation or synchronized in termittent mandatory ventilation + pressure support) were used in most cases in both ICUs, but 21 patients were ventilated in the pressure-regulated volume contro mode, with 12 in the test group and nine in the control group. The volume-controlled mode was not used. Finally, groups were similar in their use of drugs, including benzodiazepines, opioids, dobutamine, dopamine, noradrenaline, milrinone, and adrenaline (all $p$ values $>.05$ ) 
Table 2. Demographic and clinical baseline characteristics of the patients analyzed according to the study group

\begin{tabular}{lccc}
\hline \multicolumn{1}{c}{ Characteristics } & $\begin{array}{c}\text { Test Group } \\
(\mathrm{n}=134)\end{array}$ & $\begin{array}{c}\text { Control Group } \\
(\mathrm{n}=126)\end{array}$ & $p$ \\
\hline${\text { Age }(\mathrm{yrs})^{a}}^{a}$ & $1.160[0.371 ; 3.506]$ & $0.712[0.254 ; 2.172]$ & .070 \\
Pediatric Risk of Mortality II $^{a}$ & $0.042[0.023 ; 0.097]$ & $0.051[0.028 ; 0.128]$ & .419 \\
${\text { Pediatric Index of Mortality }{ }^{a}}$ & $0.091[0.033 ; 0.188]$ & $0.081[0.036 ; 0.180]$ & .598 \\
Male $^{b}$ & $71(53.0 \%)$ & $72(57.1 \%)$ & .501 \\
Immunodeficiency $^{b}$ & $4(3.0 \%)$ & $3(2.4 \%)$ & .764 \\
Congenital Heart Disease $^{b}$ & $6(4.5 \%)$ & $8(6.3 \%)$ & .504 \\
Genetic Disorders $^{b}$ & $7(5.2 \%)$ & $6(4.8 \%)$ & .864 \\
Malignant Tumors $^{b}$ & $8(6.0 \%)$ & $3(2.4 \%)$ & .151 \\
Neurologic Disorder $^{b}$ & $15(11.2 \%)$ & $9(7.1 \%)$ & .259 \\
Liver Disease $^{b}$ & $7(5.2 \%)$ & $15(11.9 \%)$ & .053 \\
Chronic Lung Disease $^{b}$ & $20(14.9 \%)$ & $17(13.5 \%)$ & .741 \\
Leukemia $^{b}$ & $4(3.0 \%)$ & $5(4.0 \%)$ & .665 \\
Main Indications for Mechanical Ventilation $^{b}$ & $3(2.2 \%)$ & $6(4.8 \%)$ & .266 \\
Congestive heart failure $^{b}$ & $34(25.4 \%)$ & $30(23.8 \%)$ & .770 \\
Wheezing $^{b}$ & $26(19.4 \%)$ & $18(14.3 \%)$ & .271 \\
Septic shock $^{b}$ & $19(14.2 \%)$ & $13(10.3 \%)$ & .344 \\
Coma $^{b}$ & $55(41.0 \%)$ & $55(43.7 \%)$ & .671 \\
Pneumonia $^{b}$ & $28(20.9 \%)$ & $32(25.4 \%)$ & .389 \\
Bronchiolitis $^{b}$ & $7(5.2 \%)$ & $6(4.8 \%)$ & .864 \\
Acute respiratory distress syndrome $^{b}$ & & & \\
\hline
\end{tabular}

Values are median [25th percentile and 75 th percentile] or numbers (percent).

${ }^{a}$ Student's $t$ test; ${ }^{b}$ Chi-square test.

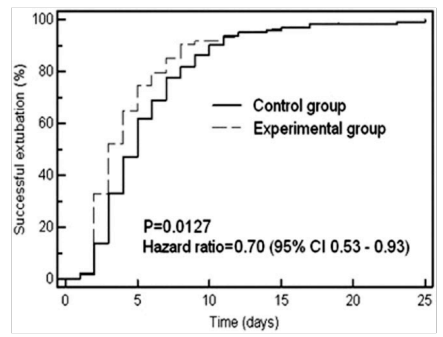

Figure 3. Comparison of the extubation time between groups.

\section{Time to Extubation}

Twenty-one patients in the test group passed the daily evaluation but failed their first SBT. Among these patients, 20 passed on a second SBT and one passed after a third SBT. A Kaplan-Meier analysis of the time to extubation showed that the median duration of MV was 3.5 days (95\% confidence interval, 2.95-4.02) in the test group, as compared with 4.7 days $(95 \%$ confidence interval, 4.08-5.34) in the control group (Fig. 3). Within the 30-day follow-up period, there was a statistically significant $30 \%$ reduction in the risk of remaining on $\mathrm{MV}$ in the test group vs. the control group (hazard ratio $=0.70 ; 95 \%$ confidence interval, $0.53-0.93 ; p=.0127$ ).

In the univariate analysis, being assigned to the test group $(p=.004)$, Pediatric Risk of Mortality II score $(p=.042)$,
Pediatric Index of Mortality 2 score $(p=$ $.024)$, liver disease $(p=.024)$, having ARDS as an indication for MV ( $p=.003)$, barotrauma $(p=.006)$, and developing ARDS $(p=.002)$ or ventilator-associated pneumonia $(p<.001)$ during MV were all factors significantly associated with the MV duration. In addition to these variables, genetic disorders $(p=.085)$, pneumonia as an indication for MV ( $p=.164)$, developing shock $(p=.077)$, ARF $(p=.067)$, and accidental extubation $(p=.129)$ showed $p$ values $<.20$ and were also included in the multivariate analysis.

In a multivariate analysis adjusting for covariates, being assigned to the test group was identified as an independent factor associated with shorter MV duration $(p<$ $.001)$, whereas the presence of ARDS as an indication for MV $(p<.001)$, and the development of ARF $(p=.018)$, ventilatorassociated pneumonia $(p<.001)$, and ARDS during the MV ( $p=.001)$ were identified as factors independently associated with longer MV duration (Table 3).

\section{Secondary Outcomes and Exploratory Analyses}

The significant reduction in the MV duration in the test group was not associated with an increase in the extubation failure rate. As presented in Table 4, an analysis of the secondary outcomes showed similar rates of reintubation, ventilator-associated pneumonia, and accidental extubation be- tween groups. Although there was a less frequent need for NIV in the test group than in the control group, this difference was not statistically sionificant ( $p=.088$ ). Importantly, comparisons between test and control groups regarding the parameters present before extubation showed no significant difference in $\mathrm{FIO}_{2}(p=.4)$, PIP ( $p=$ $.71)$, PEEP $(p=.35)$, or tidal volume $(p=$ .26), as shown in Table 5 .

Regarding the 21 patients that re quired more than one SBT, median $\mathrm{FIO}_{2}$ $\mathrm{PIP}$, and PEEP values present at the first SBT were $0.30,20 \mathrm{~cm} \mathrm{H} \mathrm{H}_{2} \mathrm{O}$, and $6 \mathrm{~cm}$ $\mathrm{H}_{2} \mathrm{O}$, respectively. In most cases, no significant changes in ventilatory parameters were observed when the first and the second SBT were compared, and the ventilatory parameters of these patients were similar to parameters shown by the rest of the sample (data not shown).

Among the 22 cases of protocol deviations, none of the five patients in the test group that were extubated without undergoing a complete SBT failed extubation and required a reintubation; of the 17 patients in the control group that were extubated after a SBT, only one failed extubation and required reintubation $(5.9 \%)$. Ventilatory parameters of these 22 patients were similar to the parameters present by the rest of the patient $\left(\mathrm{FIO}_{2}, p=.675\right.$; PIP, $p=.895$; PEEP, $p=$ 855). Furthermore, the analysis of time to extubation excluding the 22 patients who violated the protocol confirmed the overall results of the study.

In an exploratory investigation, we found that a total of 20 patients under went the first SBT with a PEEP of $8 \mathrm{~cm}$ $\mathrm{H}_{2} \mathrm{O}$, which was one of the predetermined criteria. Among these patients, only two required a second SBT. Of the 18 patients that were extubated after the first SBT, 16 were successfully extubated and only two required reintubation $(11.1 \%)$. None of the patients that performed the first SBT with PEEP of $8 \mathrm{~cm} \mathrm{H}_{2} \mathrm{O}$ died during the study course.

\section{DISCUSSION}

Although we currently have a better understanding of how to avoid MV-induced pulmonary lesions, challenges remain in reducing patient MV duration and extubating patients as early as possible ( 2 16, 27-29). Thus, it is crucial to establish strategies that can predict whether a patient is ready to be extubated. Here we show that a weaning protocol that combines a daily evaluation using pre 
Table 3. Multivariate analysis of factors predicting time to extubation

\begin{tabular}{lccr}
\hline \multicolumn{1}{c}{ Variable } & $\begin{array}{c}\text { Relative } \\
\text { Risk }\end{array}$ & $\begin{array}{c}\text { [95\% Confidence } \\
\text { Interval }]\end{array}$ & \multicolumn{1}{c}{$p$} \\
\hline Group (test) & 0.60 & {$[0.47 ; 0.78]$} & $<.001$ \\
Acute Respiratory Distress Syndrome & 3.86 & {$[2.13 ; 6.99]$} & $<.001$ \\
$\quad$ (as indication) & & & \\
Presence of Acute Renal Failure & 1.99 & {$[1.12 ; 3.52]$} & .018 \\
Ventilator-Associated Pneumonia & 3.32 & {$[2.02 ; 5.45]$} & $<.001$ \\
Acute Respiratory Distress Syndrome & 3.73 & {$[1.77 ; 7.86]$} & .001 \\
$\quad$ (as complication) & & & \\
\hline
\end{tabular}

Table 4. Respiratory complications in the two groups

\begin{tabular}{lccc}
\hline \multicolumn{1}{c}{ Variable } & $\begin{array}{c}\text { Test Group } \\
(\mathrm{n}=134)\end{array}$ & $\begin{array}{c}\text { Control Group } \\
(\mathrm{n}=126)\end{array}$ & $p$ \\
\hline $\begin{array}{l}\text { Need for Noninvasive Mechanical } \\
\text { Ventilation }^{a}\end{array}$ & $29(21.6 \%)$ & $39(31.0 \%)$ & .088 \\
Reintubation $^{a}$ & $15(11.2 \%)$ & $18(14.3 \%)$ & .454 \\
Ventilator-Associated Pneumonia $^{a}$ & $\begin{array}{c}9(6.7 \%) \\
\text { Accidental Extubation }^{a}\end{array}$ & $\begin{array}{c}12(9.5 \%) \\
8(6.3 \%)\end{array}$ & .406 \\
\hline
\end{tabular}

${ }^{a}$ Chi-square test.

Table 5. Comparison of parameters present before the extubation

\begin{tabular}{cccc}
\hline Parameter & Test Group & Control Group & $p$ \\
\hline Fio $_{2}{ }^{a}$ & & & \\
Mean $\pm \mathrm{SD}$ & $0.33 \pm 0.06$ & $0.34 \pm 0.08$ & .40 \\
Median & 0.3 & 0.3 & \\
Peak Inspiratory Pressure & & & \\
$\quad\left(\mathrm{cm} \mathrm{H}_{2} \mathrm{O}\right)^{a}$ & $18.67 \pm 2.83$ & $18.36 \pm 2.44$ & .71 \\
Mean $\pm \mathrm{SD}$ & 18.0 & 18.0 & \\
Median & & & \\
Positive End-Expiratory Pressure & $6.38 \pm 0.93$ & $6.24 \pm 0.66$ & .35 \\
$\quad\left(\mathrm{~cm} \mathrm{H}_{2} \mathrm{O}\right)^{a}$ & 6.0 & 6.0 & \\
Mean $\pm \mathrm{SD}$ & & & \\
Median & $7.36 \pm 1.94$ & $6.91 \pm 1.40$ & .26 \\
Tidal Volume $(\mathrm{mL} / \mathrm{kg})^{a}$ & 7.0 & 7.0 & \\
Mean $\pm \mathrm{SD}$ & & & \\
Median &
\end{tabular}

${ }^{a}$ Mann-Whitney $U$ test.

determined criteria with a 2 -hr SBT to assess readiness for extubation was as sociated with a shortened MV duration. This implies a reduction in the median MV duration by about 1 day as compared with standard care procedures.

Adult studies have shown that most patients do not need gradual weaning; when assessed with a daily evaluation and SBT, approximately $75 \%$ of patients are ready to be extubated $(21,23)$. Pediatric studies have shown similar results $(11$, $20)$. However, although the introduction of weaning protocols has resulted in decreased ventilation times in adult patients $(22,23)$, it is still unknown whether similar protocols can benefit the pediatric population. In a study con- ducted by Randolph et al (24), the use of a defined protocol produced no significant differences between groups with reGard to the time required for weaning. The authors found no significant differences in ventilator weaning times among the three groups (i.e., pressure support, volume support, or no protocol). They concluded that most children are weaned from MV over a short period of time and that protocols may not further shorten this brief duration. However, approximately one-third of intubated children eligible for a weaning trial actually passed an extubation readiness test, and most remained successfully extubated at 24 hrs. Thus, the question remained whether protocol-dependent weaning may indicate an earlier readiness for extubation. In our study, we showed that the application of a weaning protocol significantly shortened MV duration. Although our main finding contrasts with those presented by Randolph et al, it should be noted that both studies differ in important aspects relating to goals, study design, and methodology. One of the major differences is that in the Randolph study, only patients who failed a test for extubation readiness were randomized to one of the study groups, and therefore the test was used as a screening strategy to identify patients with more difficult weaning (24).

The MV reduction of approximately one day that was achieved with the use of the weaning protocol may not seem especially compelling at first. However, the median MV time was reduced from 4.7 to 3.5 days, which represents a reduction of almost $25 \%$ in the total time on MV. Toward that, it is also important to emphasize that although the criteria that we initially set for performing the SBT were different from those used in conventional weaning, comparative analysis showed no differences between the groups regarding the levels of PEEP, $\mathrm{FIO}_{2}$, PIP, or tidal volume before extubation. Therefore, we cannot affirm from the present study that higher ventilatory parameters can be adopted to extubate a patient earlier. On the other hand, $80 \%$ of the 20 patients that underwent a SBT with the predefined parameter originally proposed in the protocol were successfully extubated after a single SBT, thus suggesting that higher ventilatory parameters cannot be considered a risk factor for extubation failure and for the need of a second SBT. We speculate that children in the control group were probably kept under MV longer than necessary, and if the daily evaluation had been less conservative and the SBT had been performed with higher parameters we could have reduced the duration of MV even more. Importantly, all the analyses in the present study were performed on an intention-to-treat basis, and therefore the results were analyzed according to the intervention to which patients were randomized, irrespective of the treatment they had actually received during the study. One may thus speculate that the exclusion from analysis of the 22 patients that were treated differently from the group to which they were randomized could result in an even higher benefit of the test protocol. It also should be noted that subgroup analysis was not planned in our study, and therefore, we 
decided not to investigate whether our combined protocol was more successful in specific groups of children defined by age, diagnosis, or other baseline factors. Although speculative in nature, our view is that the positive results obtained in our study, in which the median age was close to $1 \mathrm{yr}$, as well as previous results in the adult population, are evidence against a treatment-by-age interaction, at least when age is considered as a possible determinant of treatment effect. In the multivariate analysis (Table 3 ), we observed that the development of ARF, ventilator-associated pneumonia, or ARDS during the ICU stay were associated with increased MV duration. However, the proportion of patients with these conditions in the two groups was similar. The association of ARF and the duration of MV contrasts with a previous study, where the cumulative fluid intake minus output was not associated with MV duration, and highlights the importance of new prospective studies in this field (30).

In the test group, there was a higher frequency of shock, which could conceivably be associated with extending times under ventilation because one of the criteria for daily evaluation was the presence of hemodynamic stability. Nevertheless, this condition was not associated with increased ventilation times in the multivariate analysis. Reassuringly, both the reintubation rate and the need for NIV were lower in the test group, albeit with no statistically significant findings.

The daily evaluations and the SBT can be easily incorporated into patient treatment routines. In our study, the SBT was applied using the pressure-support mode, which requires less time than assembling the T-piece system. We would like to stress, however, that the use of the T-piece may be preferred in places where resources are scarce.

Regardless of the system adopted for conducting the SBT, the protocol presented here remains an easy and efficient strategy to reduce pediatric patient MV duration. To our knowledge, this is the first study in which an SBT was used in pediatric intensive care patients as a weaning strategy and was performed daily until extubation. This contrasts with previous studies in which SBT was performed only in patients whose physician judged them ready to undergo a breathing trial or extubation $(6,11,20,31)$, or before randomization as in the prospective study conducted by Randolph et al (24).
The results presented above are encouraging, but our protocol has some limitations. First, we did not assess the children's upper airways because the SBT was conducted while the patients were still intubated. In addition, the open-label study design may have influenced our results, despite our attempts to minimize bias. Regarding the first limitation, it is well known that UAO is the main cause of pediatric extubation failure. In our study, 33 children failed extubation, and $57.5 \%$ had an UAO. The fact that the weaning protocol tested here was effective at shortening the MV duration but was not able to reduce the extubation failure rate highlights the importance of developing new studies in this field.

\section{CONCLUSION}

In children who received MV for $>24$ hrs, a daily evaluation to check readiness for weaning and the use of an SBT reduced MV duration without increasing the extubation failure rate or the need for NIV.

\section{ACKNOWLEDGMENTS}

We thank the dedicated residents and attending physicians in our ICUs who made this investigation possible. We also especially thank Dr. Daniel Luis Assumpcão Pitarello for his fundamental help with data collection, and Dr. Gustavo Foronda for his general support. We are grateful for the help provided by Dr. Rogério Ruscitto do Prado and the Dendrix team for their contribution to the statistical analysis.

\section{REFERENCES}

1. WHO: World health report 2005: Make every mother and child count. Geneva, World Health Organization, 2005

2. Newth CJ, Venkataraman S, Willson DF, et al: Weaning and extubation readiness in pediatric patients. Pediatr Crit Care Med 2009; $10: 1-1$

3. Tobin MJ: Advances in mechanical ventilation. $N$ Engl J Med 2001; 344:1986-1996

4. Graham AS, Kirby AL: Ventilator management protocols in pediatrics. Respir Care Clin N Am 2006; $12: 389-402$

5. Restrepo RD, Fortenberry JD, Spainhour C et al: Protocol-driven ventilator management in children: Comparison to nonprotocol care. J Intensive Care Med 2004; 19:274-284

6. Farias JA, Alía I, Retta A, et al: An evaluation of extubation failure predictors in mechanically ventilated infants and children. Intensive Care Med 2002; 28:752-757

7. Thiagarajan RR, Bratton SL, Martin LD, et al:
Predictors of successful extubation in children. Am J Respir Crit Care Med 1999; 160 1562-1566

8. Khan N, Brown A, Venkataraman ST: Predictors of extubation success and failure in mechanicully ventilated infis and childen. Crit Care ved 1996; $24: 1568-1579$ Crit Care Med 1996; 24:1568-1579

9. Venkataraman ST, Khan N, Brown A: Validation of predictors of extubation success and failure in mechanically ventilated infants and children. Crit Care Med 2000; 28:2991-2996

10. Edmunds S, Weiss I, Harrison R: Extubation failure in a large pediatric ICU population. Chest 2001; 119:897-900

11. Farias JA, Retta A, Alía I, et al: A comparison of two methods to perform a breathing trial before extubation in pediatric intensive care patients. Intensive Care Med 2001; 27: 1649-1654

12. Kurachek SC, Newth CJ, Quasney MW, et al: Extubation failure in pediatric intensive care: A multiple-center study of risk factors and outcomes. Crit Care Med 2003; 31: 2657-2664

13. Rowin ME, Patel VV, Christenson JC: Pediatric intensive care unit nosocomial infections: Epidemiology, sources and solutions. Crit Care Clin 2003; 19:473-487

14. Patel JC, Mollitt DL, Pieper P, et al: Nosocomial pneumonia in the pediatric trauma patient: A single center's experience. Crit Care Med 2000; 28:3530-3533

15. Langley JM, Bradley JS: Defining pneumonia in critically ill infants and children. Pediatr Crit Care Med 2005; 6:S9-S13

16. Bigham MT, Amato R, Bondurrant $\mathrm{P}$, et al: Ventilator-associated pneumonia in the pediatric intensive care unit: Characterizing the problem and implementing a sustainable solution. J Pediatr 2009; 154:582-587.e2

17. Gonzaga AD, Duque Figueira BB, Sousa JM, et al: [Duration of mechanical ventilation and development of bronchopulmonary dysplasia]. Rev Assoc Med Bras 2007; 5:64-67

18. Dreyfuss D, Saumon G: Ventilator-induced lung injury: Lessons from experimental studies. Am J Respir Crit Care Med 1998; 157 294-323

19. Gomes Cordeiro AM, Fernandes JC, Troster EJ: Possible risk factors associated with moderate or severe ainway injuries in who undencent endotrach diatr Crit Care Med 2004; 5:364-368

diatr Crit Care Med 2004; 5:364-368
0. Farias JA, Alía I, Esteban A, et al: Weaning from mechanical ventilation in pediatric intensive care patients. Intensive Care Med 1998; 24:1070-1075

21. Esteban A, Alía I, Tobin MJ, et al: Effect of spontaneous breathing trial duration on outcome of attempts to discontinue mechanical ventilation. Spanish Lung Failure Collaborative Group. Am J Respir Crit Care Med 1999, 159:512-518

22. Esteban A, Frutos F, Tobin MJ, et al: A comparison of four methods of weaning patients from mechanical ventilation. Spanish Lung Failure Collaborative Group. $N$ Engl J Med 1995; 332:345-350 
23. Ely EW, Baker AM, Dunagan DP, et al: Effect on the duration of mechanical ventilation of identifying patients capable of breathing spontaneously. $N$ Engl J Med 1996; 335: 1864-1869

24. Randolph AG, Wypij D, Venkataraman ST, et al: Effect of mechanical ventilator weaning protocols on respiratory outcomes in infants and children: A randomized controlled trial. JAMA 2002; 288:2561-2568

25. Twite MD: Is there a "right" way to wean my patient from the ventilator? A critical appraisal of Randolph et al: Effect of mechanical ventilator weaning protocols on respiratory outcomes in infants and children: A randomized controlled trial (JAMA 2002; 288:2561-2568). Pediatr Crit Care Med 2006; 7:571-575

26. Silva DC, Shibata AR, Farias JA, et al: How is mechanical ventilation employed in a pediatric intensive care unit in Brazil? Clinics (Sao Paulo) 2009; 64:1161-1166

27. Brower RG, Lanken PN, MacIntyre N, et al: Higher versus lower positive end-expiratory pressures in patients with the acute respiratory distress syndrome. N Engl J Med 2004 tory distress

28. Ventilation with lower tidal volumes as compared with traditional tidal volumes for acute lung injury and the acute respiratory distres syndrome. The Acute Respiratory Distress
Syndrome Network. N Engl J Med 2000; 342 : 1301-1308

29. Amato MB, Barbas CS, Medeiros DM, et at: Effect of a protective-ventilation strategy on mortality in the acute respiratory distres syndrome. N Engl J Med 1998; 338:347-35

30. Randolph AG, Forbes PW, Gedeit RG, et al: Cumulative fluid intake minus output is not associated with ventilator weaning duration or extubation outcomes in children. Pediatr or extubation outcomes in child
Crit Care Med 2005; 6:642-647

31. Chavez A, dela Cruz R, Zaritsky A: Spontaneous breathing trial predicts successful extubation in infants and children. Pediatr Crit Care Med 2006; 7:324-328 
Anexo 7 - Teste de respiração espontânea (CD)

Visualizar filme anexado ao DVD 
8 REFERÊNCIAS 
1. WHO - World Health Organization : World health report 2005: Make every mother and child count. Geneva: World Health Organization; 2005.

2. Healfaer M. Developmental physiology of the respiratory system. In Rogers M. Textbook of pediatric intensive care. Baltimore: Willians \& Wilkins; 1996. p.97-126

3. Zideman D, Hazinski M. Background and epidemiology of pediatric cardiac arrest. Pediatr Clin North Am. 2008;55(4):847-59.

4. Silva DC, Shibata AR, Farias JA, Troster EJ. How is mechanical ventilation employed in a pediatric intensive care unit in Brazil? Clinics (Sao Paulo). 2009;64(12):1161-6.

5. Tobin MJ. Advances in mechanical ventilation. N Engl $J$ Med. 2001; 344(26):1986-96.

6. Rowin ME, Patel VV, Christenson JC. Pediatric intensive care unit nosocomial infections: Epidemiology, sources and solutions. Crit Care Clin. 2003;19(3):473-87.

7. Patel JC, Mollitt DL, Pieper P, Tepas JJ 3rd. Nosocomial pneumonia in the pediatric trauma patient: A single center's experience. Crit Care Med. 2000;28(10):3530-3. 
8. Langley JM, Bradley JS. Defining pneumonia in critically ill infants and children. Pediatr Crit Care Med. 2005;6(3 Suppl):S9 -S13.

9. Bigham MT, Amato R, Bondurrant P, Fridriksson J, Krawczeski CD, Raake J, Ryckman S, Schwartz S, Shaw J, Wells D, Brilli RJ. Ventilatorassociated pneumonia in the pediatric intensive care unit: Characterizing the problem and implementing a sustainable solution. $J$ Pediatr. 2009; 154(4):582-587.e2

10. Gonzaga AD, Duque Figueira BB, Sousa JM, de Carvalho WB. Duration of mechanical ventilation and development of bronchopulmonary dysplasia. Rev Assoc Med Bras. 2007;53(1):64-7.

11. Dreyfuss D, Saumon G. Ventilator-induced lung injury: Lessons from experimental studies. Am J Respir Crit Care Med. 1998;157(1):294-323.

12. Gomes Cordeiro AM, Fernandes JC, Troster EJ. Possible risk factors associated with moderate or severe airway injuries in children who underwent endotracheal intubation. Pediatr Crit Care Med. 2004;5(4):364-8.

13. Newth CJ, Venkataraman S, Willson DF, Meert KL, Harrison R, Dean JM, Pollack M, Zimmerman J, Anand KJ, Carcillo JA, Nicholson CE; Eunice Shriver Kennedy National Institute of Child Health and Human Development Collaborative Pediatric Critical Care Research Network. Weaning and extubation readiness in pediatric patients. Pediatr Crit Care Med. 2009;10(1):1-11.

14. Graham AS, Kirby AL. Ventilator management protocols in pediatrics. Respir Care Clin N Am. 2006;12(3):389-402. 
15. Restrepo RD, Fortenberry JD, Spainhour C, Stockwell J, Goodfellow LT. Protocol-driven ventilator management in children: Comparison to nonprotocol care. J Intensive Care Med. 2004;19(5):274-84.

16. Farias JA, Alía I, Retta A, Olazarri F, Fernández A, Esteban A, Palacios K, Di Nunzio L, Fernández G, Bordón A, Berrondo C, Sheehan G. An Evaluation of extubation failure predictors in mechanically ventilated infants and children. Intensive Care Med. 2002;28(6):752-7.

17. Ravi R,, Thiagarajan RR, Bratton SL, Martin LD, Brogan TV, Taylor D. Predictors of successful extubation in children. Am J Respir Crit Care Med. 1999;160(5 Pt 1):1562-6.

18. Nadeem $\mathrm{K}$ Predictors of extubation success and failure in mechanically ventilated infants and children. Crit Care Med. 1996;24(9):1568-79.

19. Thiagarajan RR, Bratton SL, Martin LD, Brogan TV, Taylor D. Predictors of successful extubation in children. Am J Respir Crit Care Med. 1999;160(5 Pt 1):1562-6.

20. Khan N, Brown A, Venkataraman ST. Predictors of extubation success and failure in mechanically ventilated infants and children. Crit Care Med. 1996;24(9):1568-79.

21. Venkataraman ST, Khan N, Brown A. Validation of predictors of extubation success and failure in mechanically ventilated infants and children. Crit Care Med. 2000;28(8):2991-6.

22. Edmunds $\mathrm{S}$, Weiss I, Harrison R. Extubation failure in a large pediatric ICU population. Chest. 2001;119(3):897-900. 
23. Farias J, Retta A, Alía I, Olazarri F, Esteban A, Golubicki A, Allende D, Maliarchuk O, Peltzer C, Ratto ME, Zalazar R, Garea M, Moreno EG. A comparison of two methods to perform a breathing trial before extubation in pediatric intensive care patients. Intensive Care Med. 2001;27(10):1649-54.

24. Kurachek SC, Newth CJ, Quasney MW, Rice T, Sachdeva RC, Patel NR, Takano J, Easterling L, Scanlon M, Musa N, Brilli RJ, Wells D, Park GS, Penfil S, Bysani KG, Nares MA, Lowrie L, Billow M, Chiochetti E, Lindgren $B$. Extubation failure in pediatric intensive care: A multiplecenter study of risk factors and outcomes. Crit Care Med. 2003;31(11):2657-64.

25. Farias JA, Alía I, Esteban A, Golubicki AN, Olazarri FA. Weaning from mechanical ventilation in pediatric intensive care patients. Intensive Care Med. 1998;24(10):1070-5.

26. Chavez A, dela Cruz R, Zaritsky A. Spontaneous breathing trial predicts successful extubation in infants and children. Pediatr Crit Care Med. 2006;7(4):324-8.

27. Randolph AG, Wypij D, Venkataraman ST, Hanson JH, Gedeit RG, Meert KL, Luckett PM, Forbes P, Lilley M, Thompson J, Cheifetz IM, Hibberd P, Wetzel R, Cox PN, Arnold JH; Pediatric Acute Lung Injury and Sepsis Investigators (PALISI) Network. Effect of mechanical ventilator weaning protocols on respiratory outcomes in infants and children: A randomized controlled trial. JAMA. 2002; 288(20):2561-8.

28. Twite MD. Is there a "right" way to wean my patient from the ventilator? A critical appraisal of Randolph et al: Effect of mechanical ventilator weaning protocols on respiratory outcomes in infants and children: A randomized controlled trial. Pediatr Crit Care Med. 2006;7(6):571-5. 
29. Jouvet P, Farges C, Hatzakis G, Monir A, Lesage F, Dupic L, Brochard $L$, Hubert $P$. Weaning children from mechanical ventilation with a computer-driven system (closed-loop protocol): A pilot study. Pediatr Crit Care Med. 2007;8(5):425-32.

30. Moraes MA, Bonatto RC, Carpi MF, Ricchetti SM, Padovani CR, Fioretto JR. Comparison between intermittent mandatory ventilation and synchronized intermittent mandatory ventilation with pressure support in children. J Pediatr. 2009;85(1):15-20.

31. Kollef MH, Shapiro SD, Silver P, St John RE, Prentice D, Sauer S, Ahrens TS, Shannon W, Baker-Clinkscale D. A randomized, controlled trial of protocol-directed versus physician-directed weaning from mechanical ventilation. Crit Care Med. 1997;25(4):567-74.

32. Ely EW, Baker AM, Dunagan DP, Burke HL, Smith AC, Kelly PT, Johnson MM, Browder RW, Bowton DL, Haponik EF. Effect on Duration of mechanical ventilation of identifying patients capable of breathing spontaneously. N Engl J Med. 1996; 335(25):1864-9.

33. Brower RG, Lanken PN, Maclntyre N, Matthay MA, Morris A, Ancukiewicz M, Schoenfeld D, Thompson BT; National Heart, Lung, and Blood Institute ARDS Clinical Trials Network. Higher versus lower positive end-expiratory pressures in patients with the acute respiratory distress syndrome. N Engl J Med. 2004;351(4):327-36.

34. Ventilation with lower tidal volumes as compared with traditional tidal volumes for acute lung injury and the acute respiratory distress syndrome. The Acute Respiratory Distress Syndrome Network. N Engl J Med. 2000;342:1301-8. 
35. Amato MB, Barbas CS, Medeiros DM, Magaldi RB, Schettino GP, Lorenzi-Filho G, Kairalla RA, Deheinzelin D, Munoz C, Oliveira R, Takagaki TY, Carvalho CR. Effect of a protective-ventilation strategy on mortality in the acute respiratory distress syndrome. $N$ Engl $J$ Med. 1998;338(6):347-54.

36. Esteban A, Alía I, Tobin MJ, Gil A, Gordo F, Vallverdú I, Blanch L, Bonet $A$, Vázquez $A$, de Pablo $R$, Torres $A$, de La Cal MA, Macías S. Effect of spontaneous breathing trial duration on outcome of attempts to discontinue mechanical ventilation. Spanish Lung Failure Collaborative Group. Am J Respir Crit Care Med. 1999;159(2):512-8.

37. Esteban A, Frutos F, Tobin MJ, Alía I, Solsona JF, Valverdú I, Fernández R, de la Cal MA, Benito $S$, Tomás $R$, et al. A comparison of four methods of weaning patients from mechanical ventilation. Spanish Lung Failure Collaborative Group. N Engl J Med. 1995;332(6):345-350.

38. Randolph AG, Forbes PW, Gedeit RG, Arnold JH, Wetzel RC, Luckett PM, O'Neil ME, Venkataraman ST, Meert KL, Cheifetz IM, Cox PN, Hanson JH; Pediatric Acute Lung Injury \& Sepsis Investigators (PALISI) Network. Cumulative fluid intake minus output is not associated with ventilator weaning duration or extubation outcomes in children. Pediatr Crit Care Med. 2005; 6(6):642-7.

39. Ista E, Van Dijk M, Tibboel D, de Hoog M. Assessment of sedation levels in pediatric intensive care patients can be improved by using the COMFORT "behavior" scale. Pediatr Crit Care Med. 2005; 6(1):58-63.

40. Quan L, Seidel JS, editors. Pediatric advanced life support instructor's manual. Dallas: American Heart Association; 1997. 
41. Pollack MM, Ruttimann UE, Getson PR. Pediatric risk of mortality (PRISM) score. Crit Care Med. 1988; 16:1110-16.

42. Slater A, Shann F, Pearson G. PIM2: a revised version of the Paediatric Index of Mortality. Intensive Care Med. 2003; 29:278-285. 